\title{
Wirkungen der Mindestlohnregelungen in der Gebäudereinigung
}

\author{
Gerhard Bosch • Thorsten Kalina • Claudia Weinkopf
}

Angenommen: 12. August 2012 / Online publiziert: 4. September 2012

(C) Institut für Arbeitsmarkt- und Berufsforschung 2012

Zusammenfassung Die Gebäudereinigungsbranche ist mit einem hohen Anteil der Personalkosten und einer großen Vergleichbarkeit und Standardisierung der angebotenen Dienstleistungen äußerst wettbewerbsintensiv. Die statistischen Voraussetzungen für eine Evaluation der Mindestlohnregelungen waren nicht ideal, was vor allem mit einem sehr hohen Anteil von Teilzeitbeschäftigung und Minijobs zusammenhängt. Die Evaluation basiert auf einer repräsentativen Unternehmensbefragung, einer Betriebsrätebefragung, Fallstudien und mikroökonometrischen Berechnungen auf der Basis der SIAB. Es hat sich gezeigt, dass der untere Mindestlohn für die Unterhaltsreinigung die ,going rate" in der Branche ist, die ansonsten vermutlich deutlich absinken würde. Der Arbeitnehmerschutz hat sich durch eine stärkere Einhaltung der Mindestlöhne infolge der effektiven Kontrollen des Zolls, die auch als wichtiger Wettbewerbsfaktor angesehen werden, verbessert. Die Beschäftigung und das Arbeitsvolumen sind zwischen 2000 und 2008 gestiegen und die Gebäudereinigungsbranche hat ihren Marktanteil auf Kosten der Innenreinigung in anderen Branchen ausbauen können. Die mikroökonometrischen Schätzungen zu den Beschäftigungswirkungen zeigen in der Summe kei-

Für die in diesem Beitrag publizierten Ergebnisse und Schlussfolgerungen zeichnen ausschließlich die Autorinnen und Autoren verantwortlich. Sie geben nicht notwendigerweise die Position des Bundesministerium für Arbeit und Soziales wieder.

G. Bosch · T. Kalina · C. Weinkopf $(\bowtie)$

Institut Arbeit und Qualifikation, Universität Duisburg-Essen, Forsthousweg 2, 47057 Duisburg, Deutschland

e-mail: claudia.weinkopf@uni-due.de

G. Bosch

e-mail: gerhard.bosch@uni-due.de

T. Kalina

e-mail: thorsten.kalina@uni-due.de ne negativen Effekte und deuten darauf hin, dass die Aufnahme der Branche in das Arbeitnehmer-Entsendegesetz (AEntG) vor allem zu Verschiebungen von geringfügiger hin zu sozialversicherungspflichtiger Beschäftigung geführt hat.

Schlüsselwörter Mindestlohn .

Gebäudereiniger-Handwerk · Gebäudereinigung ·

Beschäftigung · Arbeitnehmerschutz · Wettbewerb Effects of minimum wages in the commercial cleaning
sector

Abstract The commercial cleaning industry is characterized by a high proportion of labour costs and a pronounced comparability and standardization of services. The statistical preconditions for the evaluation have not been ideal which is mainly due to the high proportion of part-time work and mini-jobs. The evaluation is based on a representative company survey, a survey of works counsellors, company case studies and microeconometric calculations based on SIAB. The lower minimum pay rate for indoor cleaning tends to be the "going rate" which otherwise might be undercut. Employees' protection has been improved by the more effective controls by the customs which are also assessed as very important for competition. Employment and working time volume have been increasing between 2000 and 2008 and the market share of the commercial cleaning industry has grown compared to cleaners in other industries. The microeconometric analysis reveals all in all no negative impact on employment and suggests that the inclusion of the industry into the Law on the Posting of Workers (AEntG) might have led to shifts from mini-jobs to employment covered by social security. 


\section{Einleitung}

Die Gebäudereinigung ist mit gut 915.000 Beschäftigten (Ende 2010) eine der größten Branchen in Deutschland, für die über das Arbeitnehmer-Entsendegesetz branchenspezifische Mindestlohnregelungen gelten. Im Mindestlohntarifvertrag für die gewerblichen Beschäftigten in der Gebäudereinigung sind die tariflichen Lohngruppen 1 und 6 als Mindestlöhne festgelegt. Die Lohngruppe 1 betrifft die Innenund Unterhaltsreinigung und die Lohngruppe 6 die Außenreinigung (Glas- und Fassadenreinigung).

Das Institut Arbeit und Qualifikation (IAQ), Universität Duisburg-Essen, hat die Wirkungen der Mindestlohnregelungen im Gebäudereiniger-Handwerk auf die Beschäftigung, den Schutz der Arbeitnehmer/innen und die Wettbewerbsfähigkeit der Unternehmen untersucht. Vom Auftraggeber war zunächst ein ausführliches Branchenbild gewünscht, das auf der Basis verfügbarer Brancheninformationen, statistischer Daten aus unterschiedlichen Quellen und eigenen Erhebungen Besonderheiten und Entwicklungstrends in dieser Branche herausarbeitet. Weiterhin sollten die Auswirkungen der Mindestlohnregelungen kausalanalytisch untersucht werden. Dazu bot sich der Differenzvon-Differenzen-Ansatz an, der mittlerweile zum selbstverständlichen Instrumentenkasten von Mindestlohnevaluationen gehört. Dabei werden jeweils eine Behandlungs(Treatment-) und eine damit vergleichbare Kontrollgruppe unterschieden und die Veränderungen z. B. der Löhne oder der Beschäftigung in beiden Gruppen nach einer exogenen Politikintervention in der Behandlungsgruppe miteinander verglichen. Da die Kontrollgruppe nicht von der Intervention betroffen ist, wird die Differenz auf die Wirkungen der Intervention, in diesem Fall also den Mindestlohn, zurückgeführt. ${ }^{1}$

Bei der Konzeption der Evaluation waren mehrere Besonderheiten der Branche zu beachten. Erstens haben im Unterschied $\mathrm{zu}$ den meisten anderen Branchen mit Mindestlohnregelungen - in der Gebäudereinigungsbranche allgemeinverbindliche Tarifverträge eine lange Tradition. Bereits seit Ende der 1970er Jahre sind die bis 2003 auf regionaler Ebene ausgehandelten Lohntarifverträge im Gebäudereiniger-Handwerk überwiegend für allgemeinverbindlich erklärt worden. Insofern hatten sich die Sozialpartner schon vor der Aufnahme des GebäudereinigerHandwerks in das Arbeitnehmer-Entsendegesetz (AEntG)

\footnotetext{
${ }^{1}$ Die Grenzen des Differenz-von-Differenzen-Ansatzes liegen in seinem ausschließlich mikroökonomischen Untersuchungshorizont. Mögliche gesamtwirtschaftliche Effekte wie etwa die Auswirkungen einer Erhöhung der Nachfrage werden nicht untersucht. Diese Einschränkung ist bei der Evaluation von Mindestlöhnen in kleineren Branchen ohne große gesamtwirtschaftliche Ausstrahlung hinnehmbar, schränkt aber die Aussagekraft von Evaluationen zu Mindestlöhnen mit erkennbaren Auswirkungen auf die gesamtwirtschaftliche Lohnsumme ein.
}

zum 1. Juli 2007 auf Lohnuntergrenzen geeinigt, die sich zudem auf fast das gesamte Spektrum der tariflichen Lohngruppen bezogen.

Aufgrund der Tradition von allgemeinverbindlichen Tarifverträgen konnten die Auswirkungen der Mindestlohnregelungen nach dem AEntG nicht durch einen einfachen Vorher-Nachher-Vergleich (Zustand ohne versus mit Mindestlohn) untersucht werden. Auch eine Langzeitevaluation, die die Auswirkungen der Einführung allgemeinverbindlicher Tarifverträge in der Branche einschloss, war aufgrund mehrerer Datenrevisionen nicht möglich. Von besonderem Interesse waren für die Evaluation aber mehrere Phasen, in denen sich die Bedingungen der Entlohnung verändert haben. Dies betrifft zum einen das Jahr 2004, als in der Folge des Tarifabschlusses von Herbst 2003 ab 1. April vor allem in manchen westdeutschen Regionen die nominalen Tariflöhne in Lohngruppe 1 (unterer Mindestlohn) teils deutlich abgesenkt wurden, während sie in einigen ostdeutschen Bundesländern zumindest leicht stiegen. Zum anderen könnte die Aufnahme ins Arbeitnehmer-Entsendegesetz (AEntG) zum 1. Juli 2007, obwohl damit zunächst (bis März 2008) keine Erhöhung der zuvor allgemeinverbindlichen Löhne einherging, zu einer höheren Verbindlichkeit der Mindestlöhne geführt haben. Diese Zeitpunkte wurden im Rahmen dieser Evaluation für mikroökonometrische Schätzungen zu den Beschäftigungswirkungen genutzt. Überdies war die Periode zwischen Oktober 2009 und März 2010, als die Mindestlöhne zeitweilig ausgesetzt waren, von besonderem Interesse. Da hierzu im Untersuchungszeitraum noch keine Daten vorlagen, wurde diese Phase in den Befragungen thematisiert.

Zweitens war zu berücksichtigen, dass die Mindestlohnregelungen nicht für alle Beschäftigten der Branche, sondern nur für gewerblich Beschäftigte in Betrieben gelten, die überwiegend Reinigungstätigkeiten durchführen. Es handelt sich also nicht um einen Branchenmindestlohn im eigentlichen Sinne, sondern nur für bestimmte Beschäftigtengruppen. Um den Geltungsbereich und die Auswirkungen der Mindestlohnregelungen zu bestimmen, musste daher die Gruppe der von den Mindestlohnregelungen Betroffenen innerhalb der Branche identifiziert werden. Da die überwiegende Anzahl der Beschäftigten in der Branche in der Innenund Unterhaltsreinigung (Lohngruppe 1) arbeiten, haben wir die mikroökonometrischen Analysen auf diese Gruppe konzentriert.

Drittens war zu beachten, dass Reinigungstätigkeiten nicht nur von Beschäftigten in Gebäudereinigungsunternehmen durchgeführt werden können, sondern auch intern mit eigenen Beschäftigten von Unternehmen in anderen Branchen, die keiner oder einer anderen Mindestlohnregelung unterliegen. Insofern konnte sich die Evaluation nicht alleine auf Veränderungen in der Branche selbst beschränken, sondern musste auch analysieren, ob es im Zeitablauf zu 
spürbaren Verschiebungen des Arbeitsvolumens von Reinigungskräften innerhalb und außerhalb der Gebäudereinigungsbranche gekommen ist. Diese Erweiterung des Analysehorizonts über den Geltungsbereich der untersuchten Mindestlöhne, also über die Gebäudereinigungsbranche hinaus, auf Reinigungskräfte in anderen Branchen erfolgte sowohl in den deskriptiven Analysen als auch bei den mikroökonometrischen Berechnungen.

Viertens war zu berücksichtigen, dass die Branche durch einen sehr hohen Anteil von Teilzeitarbeit und geringfügiger Beschäftigung geprägt ist. Nur etwa jede/r fünfte Beschäftigte in der Gebäudereinigungsbranche ist in Vollzeit tätig. Um Beschäftigungswirkungen feststellen zu können, musste daher auch die Entwicklung des Arbeitsvolumens bzw. der durchschnittlichen Arbeitszeit untersucht werden. Eine steigende Zahl von Beschäftigten muss nicht unbedingt mit einer Erhöhung der Zahl der geleisteten Arbeitsstunden einhergehen, sondern dahinter kann auch eine Umverteilung eines stagnierenden oder ggf. sogar sinkenden Arbeitsvolumens ,auf mehr Köpfe“" stehen.

Bei der Entwicklung des Untersuchungsdesigns für die Evaluation mussten wir von erheblichen Datenrestriktionen hinsichtlich der Genauigkeit der Daten und ihrer Zeitnähe ausgehen. Da keine Datenquelle existiert, die sowohl Angaben zum Verdienst als auch zur Arbeitszeit von Teilzeitbeschäftigten beinhaltet, mussten zur Ermittlung von Stundenlöhnen Angaben aus zwei verschiedenen Datenquellen kombiniert werden, was zwar grundsätzlich möglich ist, aber gewisse Unschärfen mit sich bringt. Es war rasch absehbar, dass kausale Wirkungsanalysen nur begrenzt möglich waren.

Unter Beachtung dieser Branchenbesonderheiten und Datenrestriktionen haben wir die Wirkungen der Mindestlohnregelungen in der Gebäudereinigung auf Beschäftigung, Arbeitnehmerschutz und Wettbewerb mit einem breiten Set unterschiedlicher methodischer Instrumente untersucht. Neben Auswertungen der amtlichen Statistik und des Mikrozensus sowie Sonderauswertungen der Bundesagentur für Arbeit zu unterschiedlichen Aspekten der Branchenentwicklung und einer Analyse vorliegender Dokumente beinhaltete das Untersuchungskonzept eine repräsentative Betriebsbefragung, eine Befragung von Betriebsräten sowie 15 vertiefende Fallstudien in ausgewählten Betrieben. Zusätzlich wurden Expertengespräche mit Vertreter/innen der Sozialpartner und der Kontrollbehörden (dem Zoll bzw. der Finanzkontrolle Schwarzarbeit) durchgeführt. Der gewählte Methodenmix sowie die Kombination von deskriptiven, qualitativen und mikroökonometrischen Analysen ermöglichte eine genaue Beschreibung der Entwicklung der Branche in den letzten Jahren, der Bedeutung und Akzeptanz der Mindestlöhne in den Betrieben und der Einschätzung unterschiedlicher Akteure über die Wirksamkeit und die Auswirkungen der Mindestlöhne auf die tatsächliche Bezahlung, die Beschäftigung, den Arbeitnehmerschutz sowie die Wettbewerbsfähigkeit. Die Verwendung von unterschiedlichen Erhebungsmethoden und Datenquellen sollte die Zuverlässigkeit der Ergebnisse erhöhen.

In diesem Beitrag werden die zentralen Ergebnisse der umfangreichen Evaluation der Wirkungen der Mindestlohnregelungen in der Gebäudereinigung (Bosch u. a. 2011) dargestellt. In Abschn. 2 geben wir zunächst einen Überblick über Struktur und Entwicklung der Branche vorrangig seit 2000. Eine besondere Zäsur war die Abschaffung der Meisterpflicht, die seit 2004 zu einem sprunghaften Anstieg der Zahl der Betriebe sowie der Markteintritte und -austritte geführt hat. An die Branchenanalyse schließt sich in Abschn. 3 eine Übersicht über die Genese der Mindestlohnregelungen in der Gebäudereinigung an. In Abschn. 4 stehen die Ergebnisse der deskriptiven Untersuchungsteile im Mittelpunkt und in Abschn. 5 die der mikroökonometrischen Analyse der Beschäftigungswirkungen. In Abschn. 6 fassen wir die Ergebnisse zusammen und geben einen Ausblick auf weiteren Forschungsbedarf.

\section{Entwicklung der Branche}

Die Gebäudereinigung ist eine bislang kaum untersuchte Branche. Die wenigen vorliegenden Analysen sind eher deskriptiv gehalten und zeigen eine hohe Wachstumsdynamik bis Anfang des Jahrtausends. Reinigungsdienstleistungen wurden seit den 1970er Jahren in zunehmendem Maße ausgelagert und an spezialisierte Dienstleister vergeben. Als Gründe für die Verlagerung werden Kompetenz- und Skalenvorteile der Konzentration von Reinigungsdienstleistungen bei spezialisierten Unternehmen, aber auch Lohnunterschiede zwischen der eigenen Reinigung und der Vergabe genannt (Grömling 2010).

Die bedeutendste Nachfragegruppe, die gewerblichen Kunden, hatte einer Branchenanalyse zufolge bereits Mitte der 1990er Jahre das traditionelle Kerngeschäft der Gebäudereinigung - die Unterhaltsreinigung zu $78 \%$ und die Fenster- und Glasreinigung sogar zu $92 \%$ - ausgelagert (Deutscher Sparkassenverlag 2009: 14). Da die Outsourcing-Quoten seitdem weiter gestiegen sind, wird mittlerweile in diesem Segment nicht mehr viel Spielraum für Neukundengeschäft gesehen. „Der Markt für Gebäudedienstleistungen ist heute ein ausgesprochener Käufermarkt, in dem ein heftiger Verdrängungswettbewerb herrscht. Marktanteile lassen sich mehr oder weniger nur auf Kosten der zahlreichen Wettbewerber gewinnen. “(Deutscher Sparkassenverlag 2009: 14) Bei Reinigungsdienstleistungen besteht ,die Wertschöpfung im Wesentlichen aus der Leistung der meist angelernten Mitarbeiter, ihrer Schulung sowie der Disposition und Organisation des Personaleinsatzes“ (Deutscher Sparkassenverlag 2009: 14) und wird dabei 
Tab. 1 Zahl der Betriebe und Umsatz im Gebäudereiniger-Handwerk nach Angaben des BIV, 1995 und 2003 bis 2010

\begin{tabular}{llllllllll}
\hline & 1995 & 2003 & 2004 & 2005 & 2006 & 2007 & 2008 & 2009 & 2010 \\
\hline Betriebe (Anzahl) & 5.494 & 6.874 & 13.143 & 19.304 & 23.692 & 28.024 & 31.262 & 34.469 & 37.636 \\
Umsatz (in Mio. $€$ ) & $6.678,5$ & $10.692,6$ & $10.569,8$ & $10.936,4$ & 11.122 & $11.402,8$ & $11.758,7$ & $11.200,7$ & $11.447,3^{\text {a }}$ \\
\hline
\end{tabular}

Quelle: Eigene Darstellung nach BIV (2011a)

${ }^{a}$ Angabe nach BIV (2012) Nach Veröffentlichung der Ergebnisse der Handwerkszählung 2008 im September 2011 hat der BIV seine Angaben zur Entwicklung der Branche angepasst und weist nunmehr nur noch die Betriebe mit einem Jahresumsatz von mindestens 17.500 € aus (BIV 2012). Die Angabe zum Umsatz 2010 ist damit nicht direkt mit den Angaben für die Vorjahre vergleichbar

in den Unternehmen selbst erbracht. Der Personalkostenanteil in dieser wenig kapitalintensiven Branche wird vom Statistischen Bundesamt für das Jahr 2008 auf ca. $72 \%$ beziffert. Der Wettbewerb um die ausgeschriebenen Aufträge erfolgt weitgehend über den Preis, was bei hoher Markttransparenz und guter Vergleichbarkeit der Angebote den starken Druck auf die Löhne erklärt.

Der Wettbewerb hat sich seit der Aufhebung der Meisterpflicht und der seit Anfang Mai 2004 geltenden Niederlassungsfreiheit für Einzelunternehmer aus den EUBeitrittsländern weiter intensiviert. Im Vergleich zu 2003 hat sich die Zahl der Betriebe bis 2010 fast verfünffacht, was offenbar vor allem auf Solo-Selbständige und andere Kleinstbetriebe zurückgeht (Tab. 1). Hierfür spricht, dass die Zahl der Unternehmen mit einem Jahresumsatz von mindestens $17.500 €$ zwischen 2003 und 2008 nach Angaben des Statistischen Bundesamtes nur um gut $21 \%$ gestiegen ist. Mit der erheblichen Zunahme der Zahl der Betriebe haben sich auch die jährlichen Markteintritte und Marktaustritte sprunghaft erhöht. Während diese in den Jahren 2002 und 2003 nur bei gut 700 Markteintritten und 500 Marktaustritten gelegen hatten, wurden im Jahr 2010 deutlich mehr als 10.000 Markteintritte und rund 7.600 Marktaustritte verzeichnet.

Parallel zu dieser zunehmenden Fluktuation am unteren Rand hat jedoch auch die Konzentration in der Branche weiter zugenommen. Auf die umsatzstarken Unternehmen mit mindestens 1 Million $€$ Jahresumsatz, die weniger als $9 \%$ aller Betriebe ausmachen, entfielen im Jahr 2008 knapp $80 \%$ aller Beschäftigten und $72 \%$ der Umsätze in der Gebäudereinigungsbranche. Für eine zunehmende Konzentration am oberen Rand spricht auch, dass nach der Dienstleistungsstatistik des Statistischen Bundesamtes der Umsatzanteil der umsatzstärksten Unternehmen zwischen 2003 und 2007 gestiegen ist. Während die 38 Unternehmen mit mindestens 25 Millionen $€$ Jahresumsatz im Jahr 2003 mit einem Anteil von 0,19\% an allen Unternehmen 18,7\% des Gesamtumsatzes der Branche erwirtschafteten, waren es im Jahr 2007 schon 22,9 \%. Die Zahl der Unternehmen mit mindestens 25 Millionen $€$ Jahresumsatz lag im Jahr 2007 mit 47 etwas höher als 2003, ihr Anteil an allen Unternehmen hatte sich jedoch mit 0,2\% kaum verändert. Da die Löhne weitgehend aus dem Wettbewerb genommen wur- den, konnten die großen Unternehmen - wie auch in vielen anderen Bereichen einfacher Dienstleistungen - ihre Overheadkosten durch Skaleneffekte senken und von den zunehmend größeren Ausschreibungen der Kunden profitieren, die ebenfalls durch die Zentralisierung ihres Einkaufs ihre Vergabekosten verringern wollten. Die polarisierte Unternehmensstruktur weist auf einen geteilten Markt mit vielen kleinen, meist nur lokal orientierten Unternehmen und wenigen größeren regional oder sogar bundesweit aufgestellten Anbietern.

Die Umsätze der Branche sind in den letzten Jahren gestiegen. Allerdings ist in den beiden Krisenjahren 2004 $(-1,1 \%)$ und vor allem $2009(-4,7 \%)$ der Umsatz zurückgegangen, was eine nicht unbeachtliche Konjunkturabhängigkeit der Branche erkennen lässt. Während im Jahr 2004 die Beschäftigtenzahl um 2,6\% und damit stärker als der Umsatz zurückgegangen ist, ist es der Branche im Jahr 2009 gelungen, die Beschäftigtenzahl trotz eines deutlich stärkeren Umsatzrückgangs stabil zu halten. Im Jahr 2010 ist die Beschäftigung nach unseren Berechnungen auf der Basis von Angaben der Bundesagentur für Arbeit sogar bereits wieder um 3,5\% gewachsen.

Zwischen 1999 und 2010 ist die Zahl der Beschäftigten in der Gebäudereinigungsbranche von knapp 665.000 auf gut 915.000 deutlich gestiegen (Abb. 1). Hierbei ist allerdings zu beachten, dass geringfügig Beschäftigte in Nebenjobs erst seit 2003 statistisch erfasst werden. Deren Zahl ist von gut 133.000 (2003) auf gut 196.000 im Jahr 2010 gestiegen.

Die Beschäftigungsstruktur ist durch einen sehr hohen Anteil von Teilzeitarbeit und insbesondere geringfügiger Beschäftigung geprägt. Nur etwa jede/r fünfte Beschäftigte ist in Vollzeit tätig (Abb. 2). Mehr als die Hälfte der Beschäftigten arbeiten in Minijobs, wobei es sich hierbei (vor allem in Westdeutschland) zunehmend häufiger um Personen handelt, die im Nebenjob geringfügig tätig sind. Der Frauenanteil an den Beschäftigten in der Gesamtbranche liegt bei gut zwei Dritteln und der Anteil der ausländischen Beschäftigten an der sozialversicherungspflichtigen Beschäftigung bei mehr als einem Viertel. Der Anteil der Beschäftigten mit Migrationshintergrund dürfte noch deutlich höher liegen. 
Abb. 1 Zahl der Beschäftigten in der Gebäudereinigung (WZ 74.7 bzw. 81.2), Deutschland gesamt sowie West- und Ostdeutschland, 1999 bis 2010. In den Angaben für 2003 bis 2010 sind geringfügig Nebenbeschäftigte enthalten. Quelle: Bundesagentur für Arbeit o. J.; eigene Darstellung und Berechnung

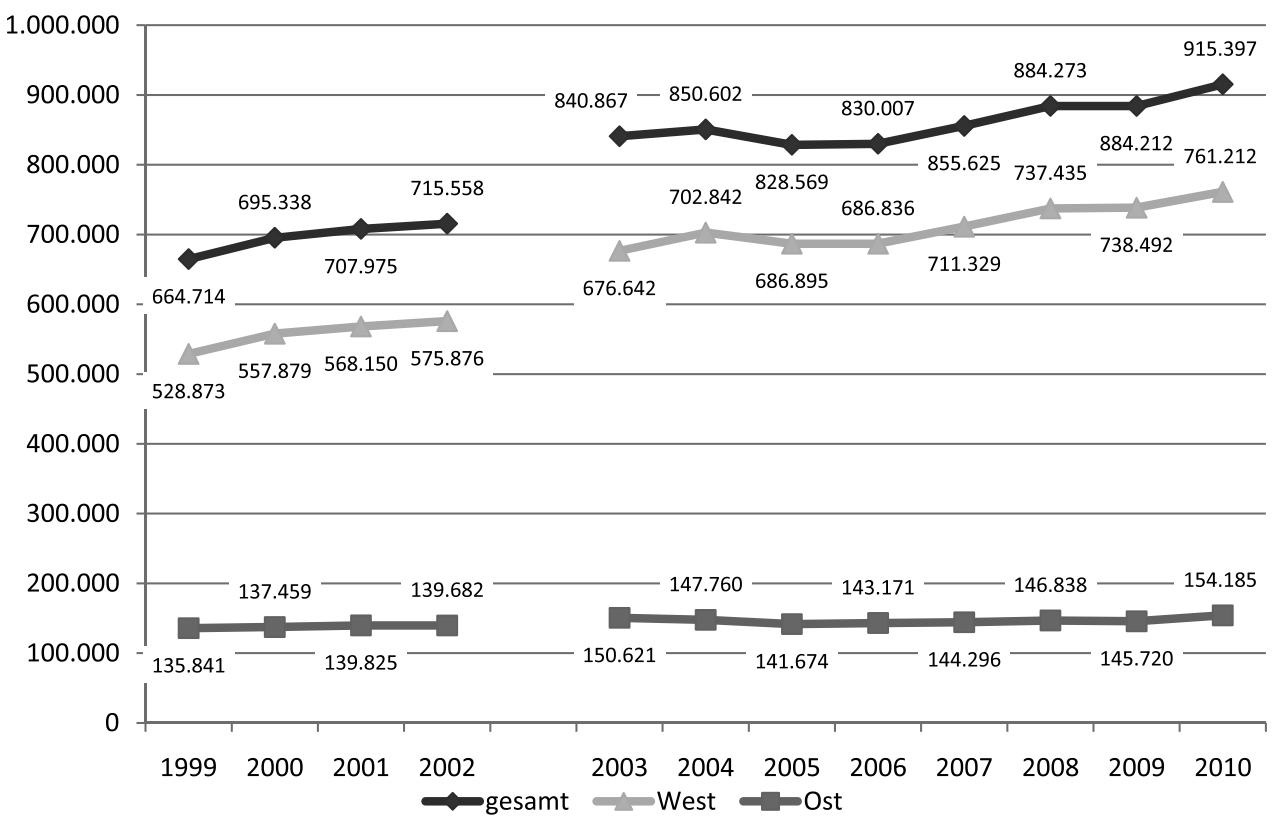

Abb. 2 Beschäftigtenstruktur in der Gebäudereinigung (WZ 81.2), Anteile verschiedener Beschäftigungsformen an der Gesamtbeschäftigung, 2003 und 2010, Deutschland gesamt sowie West- und Ostdeutschland. Quelle: Bundesagentur für Arbeit o. J.; eigene Darstellung und Berechnung

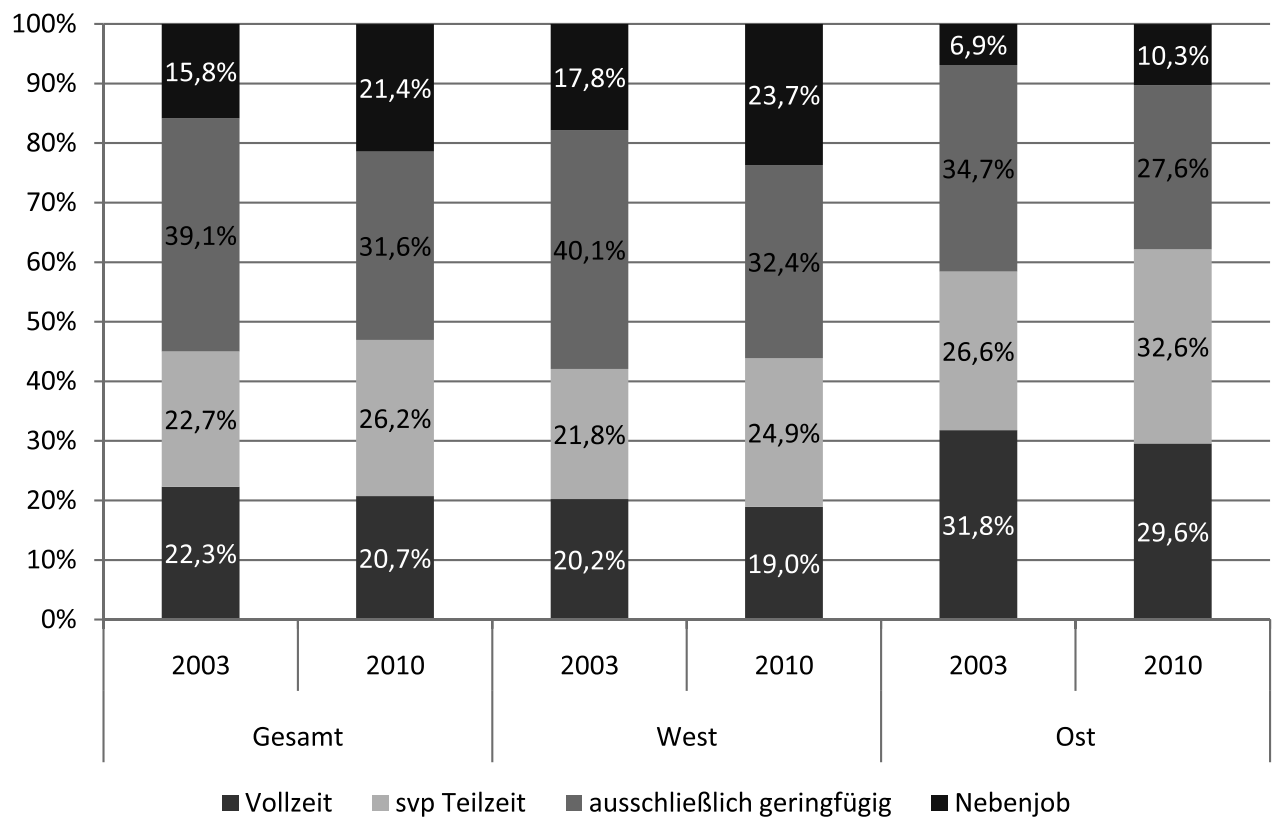

Unsere Auswertungen des Mikrozensus haben gezeigt, dass die durchschnittliche Wochenarbeitszeit der Reinigungskräfte in der Gebäudereinigungsbranche von 22,5 auf 20,6 Stunden gesunken ist. Die Arbeitszeitangaben aus dem Mikrozensus ermöglichen auch eine Berechnung des gesamten Arbeitsvolumens von Reinigungskräften innerhalb und außerhalb der Gebäudereinigungsbranche. Danach ist das Arbeitsvolumen von Reinigungskräften in anderen Branchen deutlich größer als in der Gebäudereinigungsbranche selbst, obwohl vorliegende Branchenberichte von einem hohen Grad des Outsourcings von Reinigungstätigkeiten aus anderen Branchen ausgehen. Auch die Zahl der Beschäftigten und Reinigungskräfte in der Gebäudereini- gungsbranche liegt im Mikrozensus deutlich niedriger als nach Angaben der Bundesagentur für Arbeit. Mögliche Ursache hierfür könnte zum einen die bekannte Untererfassung von geringfügig Beschäftigten im Mikrozensus sein (Körner und Puch 2009). Zum anderen ist nicht auszuschließen, dass Reinigungskräfte aus der Gebäudereinigungsbranche anderen Branchen zugeordnet werden, weil sie im Interview den Betrieb nennen, in dem sie Reinigungsleistungen erbringen. ${ }^{2}$ Dieser Widerspruch konnte im Rahmen der Evaluation

\footnotetext{
${ }^{2}$ Auf erhebliche Unschärfen bei der Kodierung der wirtschaftlichen Tätigkeiten von Betrieben oder Berufsklassifikationen in der amtlichen Wirtschaftszweigstatistik verweist auch Promberger (2011: 17).
} 
nicht aufgelöst werden, legt aber nahe, das Augenmerk vor allem auf die Veränderung des Arbeitsvolumens zu richten und weniger auf die absolute Größe des Arbeitsvolumens von Reinigungskräften innerhalb und außerhalb der Gebäudereinigungsbranche.

Abbildung 3 zeigt, dass die Zahl der Arbeitsstunden der Reinigungskräfte in der Gebäudereinigungsbranche zwischen 2000 und 2008 deutlich (um fast $25 \%$ ) gestiegen ist, während es bei den Reinigungskräften in anderen Branchen einen leichten Rückgang des Arbeitsvolumens (um 3,6 \%) gab. Insofern liefern die deskriptiven Auswertungen keine Hinweise darauf, dass es im Betrachtungszeitraum zu einer Rückverlagerung von Reinigungstätigkeiten aus der Gebäudereinigungsbranche heraus in andere Branchen gekommen ist.

\section{Tarifpolitik und Genese der Mindestlohnregelungen in der Branche}

Die Sozialpartner in der Branche - die Industriegewerkschaft Bauen-Agrar-Umwelt (IG BAU) und der Bundesinnungsverband des Gebäudereiniger-Handwerks (BIV) sind sich seit den 1970er Jahren einig, dass verbindliche Lohnuntergrenzen aufgrund der extrem hohen Personalintensität von Reinigungsdienstleistungen sowie der großen Markttransparenz und Standardisierung der angebotenen Dienstleistungen unabdingbar sind, um einen Wettbewerb, der vorrangig über die Höhe der Löhne ausgetragen wird, zu verhindern.

Bis 2003 wurden die Tarifverträge auf regionaler Ebene ausgehandelt und die Löhne für die gewerblich Beschäftigten für allgemeinverbindlich erklärt. Die Löhne variierten dabei regional sehr stark. Neben West-Ost-Unterschieden gab es auch in Westdeutschland ein deutliches Lohngefälle. In Bayern und Hessen sowie mit einem gewissen Abstand auch in Nordrhein-Westfalen wurden höhere Stundenlöhne gezahlt als in den anderen westdeutschen Bundesländern. Mit dem Tarifabschluss von Oktober 2003 wurden die zuvor auf regionaler Ebene abgeschlossenen Tarifvereinbarungen durch zentrale Vereinbarungen abgelöst, die nur noch für Ost- und Westdeutschland unterschiedliche Vergütungen beinhalten. In Folge dieses Tarifabschlusses änderten sich das Tarifgefüge und die Höhe der Löhne. Im Vergleich zu den vorherigen Tarifverträgen wurden die Tariflöhne in der Unterhaltsreinigung (Lohngruppe 1) in Westdeutschland ab 1. April 2004 überwiegend und teils deutlich abgesenkt, während sie in den meisten ostdeutschen Bundesländern angehoben wurden (Gather u. a. 2005: 30).

Hintergrund dieser besonderen tarifpolitischen Entwicklung waren die Tarifabschlüsse in der Zeitarbeitsbranche im Jahr 2003, die niedrigere Löhne als in der Gebäudereinigung vorsahen. Einige der marktführenden Gebäudereinigungsunternehmen hatten im Laufe des Jahres 2003 Tochterfirmen gegründet, um deren Beschäftigte auf der Basis eines Zeitarbeitstarifvertrags geringer entlohnen zu können. Mit der Drohung einiger großer Reinigungsfirmen, eigene Zeitarbeitsfirmen zu gründen oder externe Zeitarbeit stärker zu nutzen, um damit das höhere Tarifniveau in der Gebäudereinigung zu unterlaufen, ,wurde im Herbst 2003 mit der Industriegewerkschaft Bauen-Agrar-Umwelt (IG Bau) in bestehende Tarifverträge hinein neu verhandelt" (Gather u. a. 2005: 29). Der angedrohte Austritt großer Reinigungsfirmen aus dem Innungsverband hätte dazu führen können, dass die Voraussetzungen für die Allgemeinverbindlichkeit der Tarifverträge (Tarifdeckung von mehr als $50 \%$ der Beschäftigten) nicht mehr erfüllt gewesen wären.

Letztlich wurde erstmals in der Branche von den Tarifpartnern vereinbart, die tariflichen Bruttostundenlöhne nominal abzusenken (vgl. auch Schlese und Schramm 2004: $7 \mathrm{ff})$. In Westdeutschland lag die Lohnkürzung in der untersten Lohngruppe 1, nach der die meisten Innen- und Unterhaltsreinigungstätigkeiten vergütet werden, bei durchschnittlich 3,63\%. Die zuvor besonders gut bezahlten Beschäftigten in Bayern, Hessen und NRW mussten Lohnkürzungen zwischen $6 \%$ und fast $11 \%$ (um 0,49 bis $0,92 €$ pro Stunde) hinnehmen. In Ostdeutschland wurden hingegen die untersten Stundenlöhne mit dem neuen Tarifvertrag im Durchschnitt um 2,38 \% erhöht. Besonders hoch fiel der Zuwachs in Sachsen-Anhalt aus $(+6,74 \%)$, weil hier zuvor nur ein Stundenlohn von 5,79 € gezahlt worden war. Beschäftigte in Brandenburg-Ost und Potsdam mussten demgegenüber nur leichte Lohnkürzungen um 0,02 bzw. 0,03€ hinnehmen (Gather u. a. 2005: 30f). Die Tariflöhne in der Lohngruppe 6, nach der die meisten Außenreinigungstätigkeiten vergütet werden, wurden bundesweit abgesenkt, in Westdeutschland allerdings deutlich stärker als in den ostdeutschen Bundesländern. Darüber hinaus wurden mit dem Tarifabschluss 2004 Jahressonderzahlungen gestrichen und der tarifliche Urlaubsanspruch für Beschäftigte, die nach dem 1. Januar 2004 eingestellt wurden, von 30 auf $28 \mathrm{Ar}-$ beitstage im ersten und 29 Arbeitstage im zweiten Beschäftigungsjahr reduziert ( $\$ 14$ Rahmentarifvertrag für die gewerblich Beschäftigten in der Gebäudereinigung, gültig ab 1. April 2004).

Der Tarifvertrag für gewerbliche Beschäftigte in der Gebäudereinigung enthält insgesamt neun Lohngruppen, die nach Tätigkeiten und Qualifikationen gestaffelt sind. Im Mindestlohntarifvertrag für die gewerblichen Beschäftigten in der Gebäudereinigung sind die tariflichen Lohngruppen 1 und 6 als Mindestlöhne festgelegt. Der MindestlohnTarifvertrag definiert den betrieblichen Geltungsbereich der Mindestlohnregelungen für Betriebe und selbständige Betriebsabteilungen, die unter den Geltungsbereich des Rahmentarifvertrags für die gewerblichen Beschäftigten in der 
Abb. 3 Arbeitsvolumen von Reinigungskräften insgesamt sowie innerhalb und außerhalb der Gebäudereinigungsbranche (auf Basis der normalerweise geleisteten Arbeitszeit), in Stunden pro Woche, 2000 bis 2008. Quelle: Eigene Auswertungen mit dem Mikrozensus

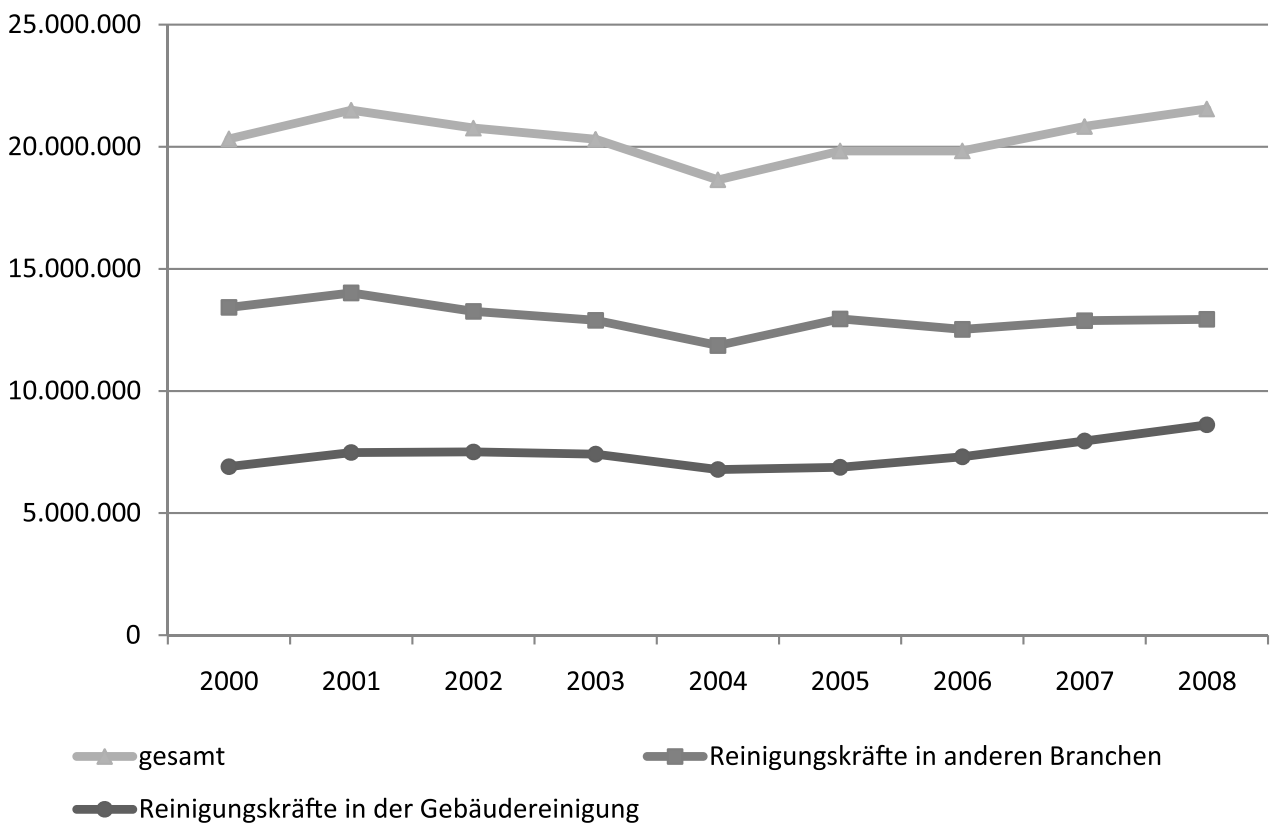

Gebäudereinigung in der jeweils geltenden Fassung fallen. Ausgenommen sind Betriebe, die überwiegend Reinigung von Verkehrs- und Freiflächen einschließlich des Winterdienstes durchführen, ,soweit dies durch Gesetz, Rechtsverordnung oder Satzung der Kommune übertragen ist“". Der persönliche Geltungsbereich der Mindestlohnregelungen umfasst gewerbliche Arbeitnehmer/innen, die sozialversicherungspflichtig oder geringfügig beschäftigt sind. Seit der Aufnahme der Branche in das ArbeitnehmerEntsendegesetz am 1. Juli 2007 gelten diese Mindestlöhne auch für Unternehmen aus dem Ausland, die grenzüberschreitende Dienstleistungen anbieten, und für Zeitarbeitskräfte, die Reinigungstätigkeiten verrichten. Zum Zeitpunkt der Aufnahme in das AEntG änderte sich die Höhe der Mindestlöhne, die seit 1. Januar 2005 tariflich vereinbart waren, zunächst nicht. Eine Erhöhung erfolgte vielmehr erst zum 1. März 2008 auf der Basis eines neuen Tarifabschlusses.

Im Zuge der konflikthaften Tarifauseinandersetzung im Jahr 2009 lief der Mindestlohntarifvertrag zum 30. September 2009 ohne Einigung über einen neuen Tarifvertrag aus und die IG BAU streikte für höhere Mindestlöhne. Da Mindestlöhne nach deutschem Recht im Unterschied zu Tarifverträgen keine Nachwirkung entfalten, kam es Ende 2009/Anfang 2010 für die nicht tarifgebundenen Unternehmen zu einem tariflosen Zustand ohne gültige Lohnuntergrenzen. Einzelne Reinigungsunternehmen boten ihren Beschäftigten nach Auslaufen der Mindestlohnregelungen Verträge zu deutlich schlechteren Konditionen an (Bispinck/WSI-Tarifarchiv 2010: 24). Mit Abschluss eines neuen Tarifvertrages waren zunächst nur die tarifgebundenen Unternehmen ab dem 1. Januar 2010 verpflichtet, die neuen Tarife zu zahlen. Die neuen Mindestlöhne traten hingegen erst nach dem entsprechenden Beschluss der Bundes- regierung am 10. März 2010 in Kraft. Weitere Tarif- und Mindestlohnerhöhungen erfolgten dann jeweils zum 1. Januar 2011 und 2012 (Tab. 2).

Dieser kurze Rückblick verweist auf eine weitere Besonderheit der Mindestlohnregelungen in der Gebäudereinigung: Über die Höhe der Mindestlöhne ist bislang immer im Rahmen der regulären Tarifverhandlungen entschieden worden und die tariflichen Lohngruppen, auf die sich die Mindestlohnregelungen beziehen, sind jeweils entsprechend der allgemeinen Steigerungsraten, auf die sich die Sozialpartner in Tarifverhandlungen geeinigt haben, erhöht worden. Spezielle Vereinbarungen zu den Mindestlöhnen wie etwa eine überproportionale Anhebung der Mindestlöhne oder gesonderte Verhandlungen über die Höhe der Mindestlöhne, wie sie in einigen anderen Branchen mit Mindestlohnregelungen üblich sind oder zumindest gelegentlich vorkommen, hat es in der Gebäudereinigung in der Vergangenheit nicht gegeben.

Dies hat sich bezogen auf die höheren Mindestlöhne für die Glas- und Außenreinigung zuletzt allerdings geändert: Die im Rahmen der letzten Tarifrunde im Sommer 2011 vereinbarten tariflichen Lohnerhöhungen in der Lohngruppe 6 für die Jahre 2012 und 2013 sind in der Mindestlohnverordnung nicht (Westdeutschland) bzw. nur teilweise (in Ostdeutschland ab Anfang 2013) berücksichtigt worden. ${ }^{3}$ Hintergrund ist offenbar, dass die Anhebung des höheren Mindestlohns entsprechend der tariflichen Erhöhungen als politisch nicht durchsetzbar angesehen wurde. So konstatiert der BIV (2011b) in einer Erklärung vom 14. November

\footnotetext{
${ }^{3}$ Die tariflichen Stundenlöhne in Lohngruppe 6 liegen in Westdeutschland seit Januar 2012 bei $11,68 €$ und steigen ab Anfang 2013 auf $11,96 €$ (Ostdeutschland: $9,32 €$ bzw. $9,79 €$ ).
} 
Tab. 2 Mindestlöhne in der Gebäudereinigung, Juli 2007 bis Oktober 2013

\begin{tabular}{lllll}
\hline Zeitraum & Lohngruppe 1 & & \multicolumn{2}{l}{ Lohngruppe 6 } \\
\cline { 2 - 4 } & Westdeutschland & Ostdeutschland & $10,43 €$ & Ostdeutschland \\
\hline $1.7 .2007-28.2 .2008$ & $7,87 €$ & $6,36 €$ & $10,80 €$ & $7,83-8,06 €$ \\
$1.3 .2008-30.9 .2009$ & $8,15 €$ & $6,58 €$ & $11,13 €$ & $7,84-8,34 €$ \\
$10.3 .-31.12 .2010$ & $8,40 €$ & $6,83 €$ & $11,33 €$ & $8,66 €$ \\
$1.1 .-31.12 .2011$ & $8,55 €$ & $7,00 €$ & $11,33 €^{\mathrm{a}}$ & $8,88 €$ \\
$1.1 .-31.12 .2012$ & $8,82 €$ & $7,33 €$ & $11,33 €^{\mathrm{a}}$ & $8,88 €^{\mathrm{a}}$ \\
$1.1 .-31.10 .2013$ & $9,00 €$ & $7,56 €$ & & $9,00 €^{\mathrm{a}}$ \\
\hline
\end{tabular}

Quelle: Eigene Zusammenstellung nach Angaben von IG BAU und BIV

${ }^{a}$ Die höheren Mindestlöhne liegen seit Anfang 2012 unter den tariflich vereinbarten Stundenlöhnen in Lohngruppe 6

2011 hierzu: „Aufgrund der politischen Situation und den Mehrheitsverhältnissen im Tarifausschuss des BMAS bestand keine Möglichkeit, die Tariferhöhung 2012/2013 der Lohngruppe 6 allgemeinverbindlich erklären zu lassen. Damit bestand nur die Alternative, die Lohngruppe 6 ganz freizugeben, oder zumindest eine Lohnuntergrenze in Höhe des Mindestlohns 2011 ,einzufrieren'. Zur Verhinderung einer sicheren Ablehnung der Allgemeinverbindlicherklärung der Lohngruppe 6 im Tarifausschuss des BMAS haben sich die Tarifparteien entschlossen, den Antrag in Höhe des bisherigen Mindestlohns 2011 zu stellen.“

\section{Deskriptive Analyse der Wirkungen}

Im Folgenden werden die deskriptiven Ergebnisse zu den Wirkungen der Mindestlöhne in der Gebäudereinigung dargestellt. Diese stützen sich neben einer Auswertung der amtlichen Statistik ${ }^{4}$ (vor allem von Daten der Bundesagentur für Arbeit und des Mikrozensus) auf drei weitere unterschiedliche Quellen. Zum ersten haben wir eine telefonische Betriebsbefragung auf der Grundlage einer nach Betriebsgröße sowie Ost- und Westdeutschland geschichteten Zufallsstichprobe von Betrieben aus dem Wirtschaftszweig 81.2, die die Bundesagentur für Arbeit aus ihrer Betriebsdatei gezogen hat, durchgeführt. Da gut $86 \%$ aller Betriebe im Wirtschaftszweig 81.2 weniger als 20 sozialversicherungspflichtig Beschäftigte haben, auf sie aber nur ein kleiner Teil der Beschäftigten entfällt, wurden größere Betriebe in der Stichprobe stärker berücksichtigt. Dies wurde durch eine Gewichtung der Befragungsergebnisse bei der Auswertung wieder ausgeglichen. Es konnten insgesamt 381 Interviews realisiert werden. ${ }^{5}$ Die Befragung ist als repräsentativ

\footnotetext{
${ }^{4}$ Die Auswertung der amtlichen Statistiken ist ausführlich erläutert in Bosch u. a. (2011: 42ff).

${ }^{5}$ Die relativ niedrige Ausschöpfung ist insbesondere einer hohen Anzahl von Schornsteinreinigungsbetrieben im Wirtschaftszweig 81.2 ge-
}

für Gebäudereinigungsbetriebe mit mindestens einem sozialversicherungspflichtig Beschäftigten anzusehen.

Zum zweiten wurde mit Unterstützung der Gewerkschaft IG BAU eine Befragung von Betriebsräten in Gebäudereinigungsbetrieben durchgeführt. Trotz dieser gewerkschaftlichen Unterstützung war der Rücklauf mit nur 42 ausgefüllten Fragebögen aus unterschiedlichen Betrieben relativ gering (knapp $10 \%$ der per E-Mail erfolgreich versandten Fragebögen). Die Ergebnisse können daher nur ein Schlaglicht auf die Sicht der betrieblichen Interessenvertretungen werfen, die zudem (erwartungsgemäß) eher in größeren Betrieben vorhanden sind. Der kleinste Betrieb im Sample hatte 70 Beschäftigte, der größte 15.000 Beschäftigte. $43 \%$ der befragten Betriebsräte gehen davon aus, dass die Arbeitsbedingungen in ihrem Betrieb besser sind als bei der Konkurrenz, während gut $38 \%$ keinen Unterschied sehen. Zum dritten wurden 15 Fallstudien in Betrieben der Gebäudereinigung durchgeführt, die sich u. a. nach Betriebsgröße, Dienstleistungsschwerpunkten und ihrem regionalen Umfeld unterschieden.

Bei der Darstellung der Ergebnisse wird zunächst nach den Wirkungen der Mindestlöhne auf die tatsächliche Bezahlung gefragt (4.1). Danach werden die Wirkungen auf Beschäftigung und Arbeitsvolumen (4.2), den Schutz der Arbeitnehmer/innen (Abschn. 4.3) und den Wettbewerb in der Branche aus Sicht der Betriebe (Abschn. 4.4) erläutert.

\subsection{Mindestlöhne und tatsächliche Bezahlung}

Da mit Hilfe der SIAB und des Mikrozensus keine präzisen Daten zur Arbeitszeit und zu den Stundenlöhnen der Beschäftigten auf der Individualebene ermittelt werden konn-

schuldet, die bei der Stichprobenziehung nicht ausgeschlossen werden konnten (knapp die Hälfte der stichprobenneutralen Ausfälle sind auf die Nicht-Zugehörigkeit zur Gebäudereinigungsbranche zurückzuführen). Die bereinigte Stichprobengröße betrug 1.713 Fälle, die Ausschöpfungsquote der realisierten Interviews an der bereinigten Stichprobe entsprach somit $22,2 \%$. 
ten, ließ sich mit Hilfe der amtlichen Statistik nicht bestimmen, welcher Anteil der Beschäftigten auf dem Niveau der beiden Mindestlöhne bezahlt wird. Auch ließ sich nicht analysieren, ob und in welchen Umfang Unterschreitungen der Mindestlöhne in der Gebäudereinigungsbranche vorkommen. Aussagen zur Einhaltung der Mindestlöhne konnten somit nur auf der Basis unserer beiden Befragungen von Betrieben und Betriebsräten sowie den Experteninterviews in Fallstudienbetrieben und beim Zoll gewonnen werden.

Obwohl die Aufnahme der Branche in das AEntG zunächst formal nicht zu Lohnerhöhungen geführt hat, weil die selben Löhne zuvor bereits (als Tariflöhne) allgemeinverbindlich (und damit prinzipiell bindend) waren, gingen die Sozialpartner wie auch die meisten Gesprächspartner/innen in den Fallstudienbetrieben davon aus, dass sich die Einhaltung der Mindestlöhne verbessert habe. Dies wurde vor allem auf die im Vergleich zur vorherigen Allgemeinverbindlichkeit effektiveren Kontrollen und Sanktionen zurückgeführt, aber auch auf die durchgreifende Auftraggeberhaftung ${ }^{6}$ sowie einen besseren Informationsstand der Beschäftigten. Allerdings konnte nicht beziffert werden, in welchem Umfang nach der Aufnahme in das AEntG tatsächlich Lohnerhöhungen vorgenommen werden mussten. In den 15 Fallstudienbetrieben waren die Beschäftigten schon zuvor mindestens auf dem Niveau der Mindestlöhne bezahlt worden.

In der Betriebsbefragung gaben gut $36 \%$ der befragten Betriebe an, dass sich die Einhaltung der Mindestlöhne nach der Aufnahme der Branche in das ArbeitnehmerEntsendegesetz verbessert habe. Die Mehrheit der Befragten (gut $54 \%$ ) vermuteten allerdings, dass sich wenig geändert habe, und $10 \%$ meinten, dass sich die Einhaltung verschlechtert habe. Bei einer weiteren Frage nach positiven Wirkungen der Mindestlohnregelungen, die Mehrfachnennungen erlaubte und nur denjenigen Betrieben gestellt wurde, die zuvor angegeben hatten, dass sie überwiegend positive oder negative Wirkungen sahen, verwiesen knapp $63 \%$ der Betriebe darauf, dass die Mindestlöhne durch die häufigeren Zollkontrollen effektiver durchgesetzt würden. In der Betriebsrätebefragung gaben knapp $43 \%$ der Befragten an, dass sich die Einhaltung der Mindestlöhne durch das AEntG verbessert habe, und mehr als $71 \%$ sahen in den Kontrollen durch den Zoll ein effektives Instrument zur besseren Durchsetzung der Mindestlöhne.

Die Betriebsbefragung hat auch die Einschätzungen der Sozialpartner bestätigt, dass die Mindestlöhne in der Gebäudereinigung eine hohe Bedeutung für die tatsächliche Entlohnung haben. Gut $45 \%$ der befragten Betriebe gaben

\footnotetext{
${ }^{6}$ Bei Vergaben, die so niedrige Vergütungen vorsehen, dass eine Unterschreitung der Mindestlöhne anzunehmen ist, können auch die Auftraggeber sanktioniert werden. Zur Frage, welche Stundenverrechnungssätze unter Berücksichtigung der Mindestlöhne realistisch sind, liegen mehrere Handlungshilfen für Auftraggeber und Reinigungsbetriebe vor (vgl. z. B. BIV 2007 und 2011c).
}

an, dass alle Reinigungskräfte auf dem Niveau der Mindestlöhne bezahlt werden, und weitere gut $29 \%$ der Betriebe sagten, dass dies zumindest bei einem Teil ihrer Reinigungskräfte der Fall sei, während die anderen höher bezahlt würden. Knapp $26 \%$ der befragten Betriebe gaben an, dass bei ihnen alle Reinigungskräfte einen über dem unteren Mindestlohn liegenden Stundenlohn erhalten. Mit Ausnahme dieser Gruppe wurden die Betriebe auch gefragt, wie hoch der Anteil der Beschäftigten ist, die nach Lohngruppe 1, also dem unteren Mindestlohn bezahlt werden. Gut $58 \%$ der Betriebe gaben hier Anteile von 80 bis $100 \%$ an und weitere 18,4\% Anteile von 60 bis $80 \%$ (Abb. 4). Die Lohngruppe 6 hat angesichts des deutlich kleineren Anteils von Reinigungskräften im Bereich der Glas- und Außenreinigung erwartungsgemäß eine wesentlich geringere Bedeutung. In mehr als der Hälfte der Betriebe liegt der Anteil der Beschäftigten in Lohngruppe 6 nach eigenen Angaben bei unter $5 \%$ und bei weiteren knapp $15 \%$ der Betriebe zwischen 5 und $10 \%$. Nur gut jeder zehnte Betrieb hatte nach eigenen Angaben einen Beschäftigtenanteil von $40 \%$ oder mehr in der Lohngruppe 6. Unsere Fallstudien weisen in die gleiche Richtung und haben gezeigt, dass die beiden Mindestlöhne in den meisten Betrieben der Gebäudereinigung als die „going rate“ gelten, die bei guter Arbeitsmarktlage z. T. leicht überschritten wird.

Der Zoll hat im Rahmen einer Schwerpunkt-Kontrolle im November/Dezember 2010 insgesamt 2.051 Gebäudereinigungsbetriebe überprüft und dabei in etwa einem Drittel der Fälle Unregelmäßigkeiten festgestellt (Zoll 2010). Anhaltspunkte für mögliche Mindestlohnverstöße wurden in $13 \%$ der geprüften Betriebe gefunden (im Vergleich zu $17 \%$ der geprüften Betriebe im Jahr zuvor).

Alle befragten Fallstudienbetriebe zahlten nach eigenen Angaben mindestens die Mindestlöhne. Dies können sie nach ihrer Einschätzung aufgrund der Wettbewerbsneutralität der Mindestlöhne, die durch die starken Kontrollen gesichert sei. Mehrere Gesprächspartner/innen vermuteten, dass die unteren Löhne ohne Mindestlohnpflicht auf 5 bis 6 $€$ fallen würden. Die Interviews zeichneten das Bild einer Branche, in der ohne gesetzliche Mindestlohnpflicht die hohe Preiskonkurrenz sofort zu Lohnkürzungen führen würde. Hervorgehoben wurde auch das verbesserte Informationsniveau der Beschäftigten, die die Mindestlöhne kennen und auch einfordern, wodurch ihre Verhandlungsmacht gestärkt worden sei.

\subsection{Beschäftigung und Arbeitsvolumen}

Vor 2003 wurden geringfügig Nebenbeschäftigte von der Bundesagentur für Arbeit statistisch nicht erfasst. Um die Wirkungen der allgemeinverbindlichen Tarifverträge und der Mindestlöhne zu beschreiben, kann daher nur der Zeitraum zwischen 2003 und 2010 sinnvoll betrachtet werden. 
Abb. 4 Anteil der nach Lohngruppe 1 bezahlten Reinigungskräfte im Betrieb (in \% der Betriebe, die angegeben haben, dass alle oder ein Teil der Reinigungskräfte auf dem Mindestlohnniveau bezahlt werden) $n=249$.

*Anteile, die auf Fallzahlen zwischen 10 und unter 20,

**Anteile, die auf Fallzahlen unter 10 basieren.

Quelle: IAQ-Betriebsbefragung Gebäudereinigung, gewichtete Werte

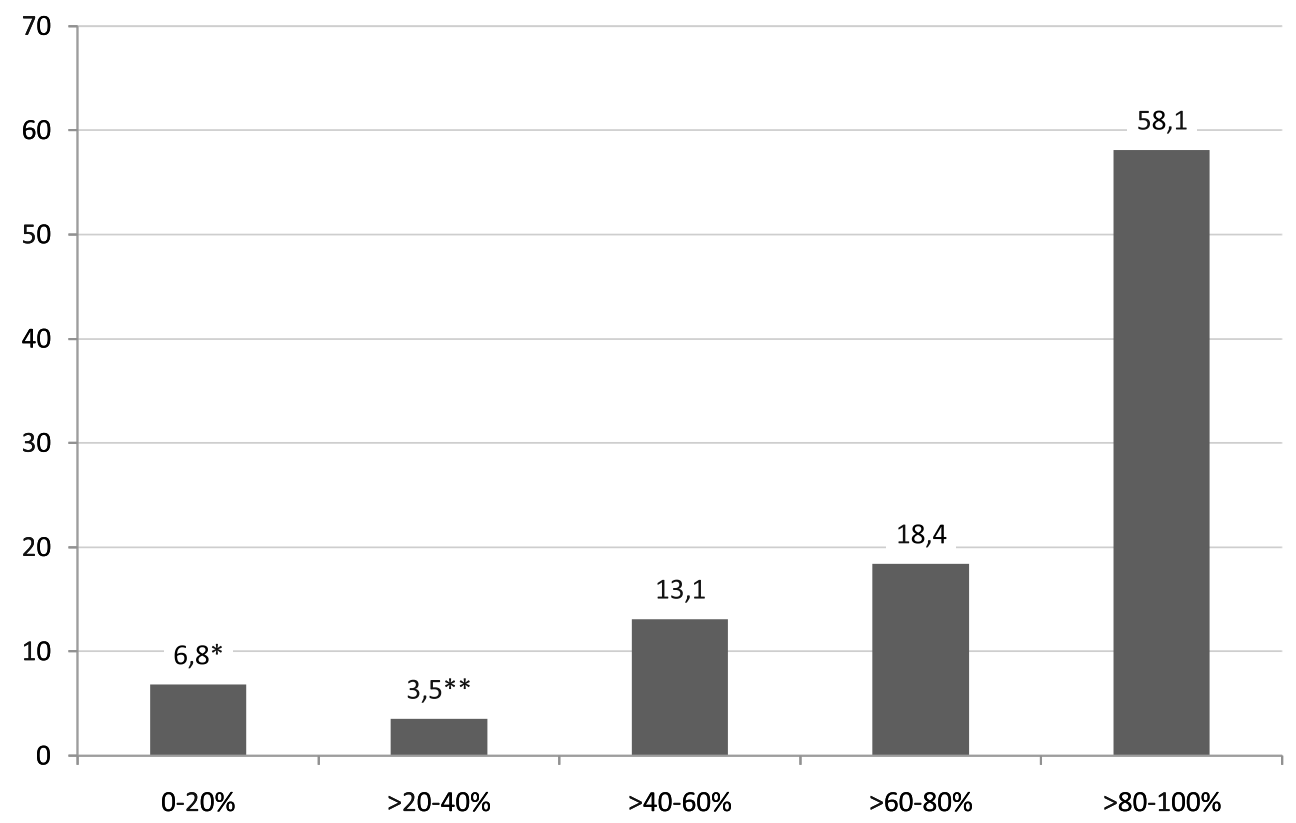

Die bereits in Abschn. 2 dargestellten Entwicklungen der Zahl der Beschäftigten und des Arbeitsvolumens zeigen keine negativen Effekte der Aufnahme des GebäudereinigerHandwerks in das AEntG und der folgenden Erhöhung der Mindestlöhne. Erkennbar sind nur konjunkturelle Schwankungen. Die Zahl der Beschäftigten ist in diesem Zeitraum um fast $9 \%$ gestiegen (Abb. 1). Dieser Zuwachs geht fast ausschließlich auf eine Zunahme der sozialversicherungspflichtig Beschäftigten zurück, deren Zahl zwischen 2005 und 2010 um gut $22 \%$ gewachsen ist. Die Zahl der geringfügig Beschäftigten stagniert demgegenüber seit 2004. Ihr Anteil an der Gesamtbeschäftigung ist in diesem Zeitraum von $57,5 \%$ auf $53 \%$ zurückgegangen. Die Zahl der ausschließlich geringfügig Beschäftigten nahm seit 2004 um fast $16 \%$ $a b$, während geringfügig Nebenbeschäftigte deutlich an Bedeutung gewonnen haben. Ihre Zahl ist zwischen 2003 und 2010 um $47 \%$ gestiegen. Im Jahr 2010 war damit gut jede/r fünfte Beschäftigte in der Gesamtbranche in einem geringfügigen Nebenjob tätig. Auch das Arbeitsvolumen nahm seit dem konjunkturellen Tief in den Jahren 2003/2004 bis 2008 kontinuierlich zu. In diesem Zeitraum konnte sich die Gebäudereinigungsbranche auf Kosten der Reinigung in anderen Branchen einen wachsenden Teil des Auftragsvolumens sichern (Abb. 3). Leider reichten die verfügbaren Daten des Mikrozensus zum Analysezeitpunkt nur bis 2008, so dass keine Aussagen zur Entwicklung am aktuellen Rand möglich waren.

Auch die Befragungen von Betrieben und Betriebsräten sowie Fallstudien und Expertengespräche, mit denen auch noch die Trends bis zum ersten Halbjahr 2011 eingefangen werden konnten, lieferten keinerlei Hinweise auf Beschäftigungsverluste. In der Betriebsbefragung wurden die Betriebe nach der Entwicklung ihrer Beschäftigung, ihres Ar- beitsvolumens und ihres Umsatzes im Vergleich zu 2007 also dem Jahr der Aufnahme der Branche in das AEntG gefragt. Falls die Betriebe erst nach 2007 gegründet worden waren, sollten sie Auskunft über die Entwicklung seit der Gründung geben. Die Beschäftigung ist nach den Befragungsergebnissen bei knapp $53 \%$ der Betriebe in den letzten Jahren (im Vergleich zu 2007) gestiegen und in weiteren gut $29 \%$ in etwa stabil geblieben. Nur gut $18 \%$ der Betriebe berichteten von rückläufiger Beschäftigung. Beim Arbeitsvolumen lagen die Anteile derjenigen, die angaben, dass dieses gestiegen (gut $54 \%$ ) oder konstant geblieben sei (32\%), noch etwas höher. Entsprechend ist das Arbeitsvolumen in weniger als $14 \%$ der Betriebe gesunken. Der Umsatz hat bei mehr als $59 \%$ der befragten Betriebe zugenommen und ist bei weiteren gut $24 \%$ in etwa stabil geblieben, während gut $16 \%$ der Betriebe einen sinkenden Umsatz verzeichneten. Differenziert nach Ost- und Westdeutschland lagen die Anteile der Betriebe mit positiver oder stabiler Entwicklung im Osten jeweils etwas höher.

In der Betriebsrätebefragung gaben mehr als $83 \%$ der Betriebsräte an, dass die Beschäftigung in ihrem Betrieb seit 2007, als die Branche in das AEntG aufgenommen worden ist, gestiegen oder in etwa gleich geblieben sei, während nur knapp $12 \%$ der Befragten von einem Rückgang der Beschäftigtenzahl berichteten (und knapp $5 \%$ hierzu keine Angabe machten). In den Betriebsfallstudien wurden von den Gesprächspartner/innen keine negativen Auswirkungen der Aufnahme der Branche in das ArbeitnehmerEntsendegesetz auf das Beschäftigungsniveau gesehen. Als Ursachen für Personalaufbau und -abbau wurden überwiegend konjunkturelle Einflüsse und die erfolgreiche Akquise neuer oder der Verlust bestehender Aufträge angeführt. 


\subsection{Arbeitnehmerschutz}

Die Einführung von Mindestlöhnen zielt u. a. darauf ab, Beschäftigte vor unangemessen niedriger Entlohnung zu schützen. Gleichzeitig kann die Beschäftigungsqualität aber auch leiden, wenn zur Kompensation der Einführung von Mindestlöhnen andere Lohnbestandteile oder freiwillige Sozialleistungen gekürzt werden oder sich andere Arbeitsbedingungen verschlechtern. Dabei ist vor allem an eine Intensivierung der Arbeit oder eine Zunahme instabiler Vertragsformen wie befristeter Beschäftigungsverhältnisse zu denken. Solche Veränderungen der Beschäftigungsqualität sind allerdings den Mindestlöhnen nur dann zuzurechnen, wenn Trendbrüche im Zusammenhang mit der Einführung oder Erhöhung von Mindestlöhnen erkennbar sind.

Der Schutz vor unangemessen niedrigen Löhnen ist durch die Mindestlohnregelungen in der Gebäudereinigungsbranche weitgehend erreicht worden. Dazu haben in der Vergangenheit bereits allgemeinverbindliche tarifliche Löhne beigetragen, wobei allerdings übereinstimmend davon ausgegangen wird, dass die Einhaltung und Durchsetzung der Mindestlöhne seit der Aufnahme in das Arbeitnehmer-Entsendegesetz noch deutlich effektiver ist. Der Arbeitnehmerschutz in der Gebäudereinigung ist auch insofern als besonders hoch zu erachten, als - anders als in den meisten anderen Mindestlohnbranchen - die große Mehrheit der Beschäftigten auf dem Niveau der unteren Mindestlöhne bezahlt wird. Mit den Mindestlöhnen wird in der Gebäudereinigungsbranche also nicht ein differenziertes Tarifgefüge stabilisiert, sondern vor allem die unterste Lohngruppe des Tarifgitters. Nach Einschätzung der Sozialpartner, Betriebsräte und auch vieler Gesprächspartner/innen in den Fallstudienbetrieben würden bei einem Wegfall der Mindestlohnregelungen die Löhne deutlich sinken.

Unsere Auswertung des Mikrozensus hat gezeigt, dass sich die durchschnittliche Wochenarbeitszeit von geringfügig Beschäftigten zwischen 2000 und 2008 deutlich erhöht hat. Unter dem Blickwinkel des Arbeitnehmerschutzes ist dies eher positiv zu bewerten, weil bei längeren Arbeitszeiten auch die monatlichen Verdienste höher ausfallen. Allerdings lag in Westdeutschland die durchschnittliche Wochenarbeitszeit der geringfügig Beschäftigten im Jahr 2008 mit 11,5 Stunden so hoch, dass bei der damaligen Höhe des westdeutschen Mindestlohnes $(8,15 €)$ jedenfalls bei ganzjähriger Beschäftigung in diesem Stundenvolumen die monatliche Geringfügigkeitsgrenze von $400 €$ leicht überschritten worden wäre. Möglicherweise sind in diesen Fällen Ansprüche z. B. auf Lohnfortzahlung bei Krankheit oder Urlaub nicht vollständig gewährt und/oder Mindestlöhne unterschritten worden. Solche Abweichungen von den Mindestlohnregelungen hätte man nur über eine Beschäftigtenbefragung identifizieren können, die im Rahmen der Evaluation nicht durchgeführt werden konnte.
Auch die befragten Betriebsräte gaben an, dass die letzten Tariflohn- und Mindestlohnerhöhungen Anfang 2011 weit überwiegend zu entsprechenden Lohnerhöhungen geführt haben. Allerdings berichteten einige Betriebsräte auch von Verkürzungen der Arbeitszeit bei einigen oder sogar allen Reinigungskräften im Betrieb. Fast $60 \%$ der Betriebsräte gaben an, dass die gleiche Fläche nun in kürzerer Zeit gereinigt werden musste, und gut ein Viertel der Befragten verwiesen darauf, dass Einsatzzeiten verändert wurden, um Zuschläge zu sparen. Ob solche Veränderungen sich negativ auf den Schutz der Beschäftigten auswirken oder nicht, lässt sich nicht pauschal beurteilen. Eine Verkürzung der Arbeitszeit in Folge von Mindestlohnerhöhungen kann auch auf Wunsch der Beschäftigten erfolgen, weil diese z. B. die Geringfügigkeitsgrenze nicht überschreiten wollen. Wenn das zu leistende Arbeitspensum entsprechend reduziert wird, arbeiten die Beschäftigten nach Mindestlohnerhöhungen weniger für das gleiche oder ein ggf. nur leicht erhöhtes Monatsentgelt. Wenn es jedoch keine Reduzierung des Arbeitspensums gibt, wie die Einschätzung der Mehrheit der Betriebsräte nahelegt, dann ist für den Arbeitnehmerschutz von Bedeutung, ob dies in der bezahlten Arbeitszeit möglich ist oder unbezahlte Mehrarbeit geleistet bzw. ggf. sogar vom Arbeitgeber eingefordert wird. Letzteres wäre ein klarer Verstoß gegen die Mindestlohnregelungen, der aber ggf. schwierig aufzudecken ist. Wenn Einsatzzeiten verändert werden, um Zuschläge einzusparen, kann dies einerseits zu einer Reduzierung des Monatsentgeltes führen, aber andererseits auch dazu, dass Reinigungskräfte seltener nachts oder an Sonn- und Feiertagen arbeiten müssen. Ob dies als Verschlechterung oder als Entlastung anzusehen ist, lässt sich nicht pauschal beurteilen, sondern hängt vor allem von den persönlichen Lebensumständen der Beschäftigten ab.

In den Fallstudienbetrieben gingen die meisten Gesprächspartner/innen davon aus, dass die Möglichkeiten einer zunehmenden Arbeitsverdichtung in der Gebäudereinigung weitestgehend ausgeschöpft seien. Sie vertraten also die Auffassung, dass Lohnerhöhungen (jedenfalls legal) nicht mehr durch entsprechende Verkürzungen der Arbeitszeit kompensiert werden könnten. Allerdings wurde berichtet, dass Auftraggeber teilweise versuchen, die Kosten für einen bestimmten Auftrag trotz Lohnerhöhungen für die Beschäftigten konstant zu halten, indem Reinigungsintervalle entsprechend angepasst werden.

\subsection{Wettbewerb aus Sicht der Betriebe}

Die Auswirkungen auf die Beschäftigung, die unmittelbar mit dem Wettbewerb in der Branche verbunden sind, sind bereits in Abschn. 4.2 beschrieben worden, so dass hier die anderen Wettbewerbsfaktoren thematisiert werden.

Bundesweit schätzten fast $60 \%$ der befragten Betriebe die Wirkungen der Mindestlöhne auf die Wettbewerbsfähig- 
keit als überwiegend positiv ein. Mit gut $61 \%$ sahen deutlich mehr Befragte in Westdeutschland positive Auswirkungen als in Ostdeutschland (gut $47 \%$ ), wo zu einem größeren Teil keine nennenswerten Wirkungen angenommen wurden. Die (wenigen) Betriebe mit Personalrückgang bewerteten die Wirkungen der Mindestlohnregelungen deutlich häufiger negativ als Betriebe mit Personalanstieg bzw. konstanten Beschäftigtenzahlen.

Bei der Frage nach wichtigen Wettbewerbsfaktoren nannten die Betriebe in der Betriebsbefragung fast gleichauf Preis und Zuverlässigkeit (mit jeweils rund $70 \%$ hoher Bedeutung) und $63 \%$ auch die Qualität, während der Breite des Dienstleistungsangebots seltener eine hohe Bedeutung beigemessen wurde (gut $24 \%$ ). Am stärksten an Bedeutung gewonnen hat in den letzten Jahren nach Angaben der Betriebe der Preis, was möglicherweise im Zusammenhang damit steht, dass fast zwei Drittel der Betriebe an anderer Stelle der Befragung angaben, dass es in den letzten Jahren eher noch schwieriger geworden sei, Kostensteigerungen (z. B. durch Lohnerhöhungen) über Preiserhöhungen an die Kunden weiter zu geben. Die größten Schwierigkeiten wurden diesbezüglich bei öffentlichen Auftraggebern bzw. in der Unterhaltsreinigung gesehen.

Mehr als $36 \%$ der befragten Betriebe meinten, dass die Aufnahme der Gebäudereinigungsbranche in das Arbeitnehmer-Entsendegesetz zu einer stärkeren Einhaltung der Mindestlöhne beigetragen habe. Gut $54 \%$ sahen keine Veränderung und knapp $10 \%$ gaben an, dass sich die Einhaltung eher verschlechtert habe. Über $44 \%$ der befragten Betriebe schätzen die Wirkungen des AEntG insgesamt als eher positiv ein, während rund $17 \%$ überwiegend negative Wirkungen sahen und knapp $39 \%$ keine nennenswerten Wirkungen. Westdeutsche und größere Betriebe schätzten die Wirkungen insgesamt positiver ein als ostdeutsche bzw. kleinere Betriebe. Als einzelne positive Wirkung nannten die Betriebe am häufigsten, dass die Mindestlöhne durch die häufigeren Zollkontrollen effektiver durchgesetzt würden (knapp $63 \%$ ) und dass das Image der Branche durch die Mindestlöhne gefördert werde (gut $60 \%$ ), wobei hier Mehrfachnennungen möglich waren. Eine positive Wirkung auf die Personalrekrutierung und -bindung wurde demgegenüber nur von $27 \%$ der Befragten genannt. Keine positiven Wirkungen sahen nur knapp $11 \%$ der Betriebe. In den Betriebsfallstudien wurden positive Wirkungen auf die Personalrekrutierung häufiger thematisiert.

Dass es keine negativen Wirkungen gebe, gaben mit über $53 \%$ deutlich mehr als die Hälfte der Betriebe an. Wenn in der Betriebsbefragung überhaupt negative Wirkungen genannt wurden, dann am ehesten eine Reduzierung der Gewinnmargen (gut $35 \%$ ), während ein notwendiger Personalabbau nur von gut $14 \%$ der Befragten angesprochen wurde.

Obwohl in den Expertengesprächen bei den Sozialpartnern und teilweise auch in den Betriebsfallstudien darauf verwiesen wurde, dass die Mithaftung der Auftraggeber ein wichtiger Faktor sei, der die Einhaltung der Mindestlöhne nach Aufnahme in das AEntG deutlich verbessert habe, wurde in der Betriebsbefragung deutlich, dass viele Betriebe bei der Weitergabe von Kostensteigerungen an die Kunden noch Verbesserungsbedarf sehen: Dass die Stundenverrechnungssätze in den vergangenen Jahren angemessen erhöht werden konnten, verneinten etwa die Hälfte der Betriebe. Nur gut ein Viertel der Betriebe berichteten von angemessenen Erhöhungen und etwas weniger Betriebe gaben an, dass die Stundenverrechnungssätze zwar erhöht werden konnten, aber in zu geringem Maße. Eine Differenzierung nach Betriebsgröße zeigte hier, dass Betriebe mit 100 oder mehr Beschäftigten deutlich erfolgreicher dabei waren, Preissteigerungen bei den Kunden durchzusetzen, als kleinere Betriebe.

Im Zuge der erweiterten Arbeitnehmerfreizügigkeit seit Mai 2011 erwarteten mehr als $41 \%$ der Betriebe, dass die Mindestlohnregelungen zur Verhinderung von Billiglohnkonkurrenz beitragen werden, während $36 \%$ eher geringe Wirkungen erwarteten, weil die Kontrolle schwierig sei. Nur gut jeder fünfte Betrieb war der Auffassung, dass die Mindestlohnregelungen in diesem Kontext keine Rolle spielen würden. Die Interviews im Rahmen der Betriebsfallstudien zeigten allerdings, dass die Gesprächspartner/innen noch keine konkreten Erfahrungen mit Arbeitnehmerentsendungen hatten.

\section{Mikroökonometrische Analysen der Beschäftigungswirkungen}

In den deskriptiven Analysen waren keine negativen Auswirkungen der Aufnahme der Gebäudereinigung in das AEntG und der folgenden Erhöhungen der Mindestlöhne zu erkennen. Allerdings können sich selbst hinter einer positiven Entwicklung der Beschäftigung und des Arbeitsvolumens negative Wirkungen verbergen, weil durchaus denkbar ist, dass die positiven Effekte ohne Mindestlöhne noch ausgeprägter gewesen wären. Solche Effekte lassen sich durch den Differenz-von-Differenzen-Ansatz identifizieren. In diesem quasi-experimentellen Ansatz vergleicht man die Beschäftigungsentwicklung in der Gebäudereinigungsbranche (Treatment-Gruppe) mit vergleichbaren Kontrollgruppen. Statistisch signifikante Unterschiede in der Ausprägung bestimmter Merkmale werden auf die exogene Politikintervention zurückgeführt und oft als „,kausal“ oder „Wirkung“ interpretiert. „Kausalität“ oder „Wirkung“ lassen sich jedoch wissenschaftlich nicht eindeutig feststellen. Auf eine gebotene Vorsicht in der Interpretation müssen wir hinweisen, da sich anders als in den Naturwissenschaften in den Gesellschaftswissenschaften Treatment- und Kontrollgruppen niemals völlig gleichen. Man kann nur versuchen, die unbeobachtete Heterogenität so weit wie möglich zu reduzieren. 
Die Gebäudereinigung ist im Unterschied zu anderen wissenschaftlich relativ gut erforschten Branchen noch nicht so umfassend untersucht worden. Damit sind möglicherweise noch nicht alle Einflussfaktoren, die Beschäftigung, Wettbewerbsfähigkeit und Arbeitnehmerschutz in dieser Branche beeinflussen, ausreichend erkannt und präzise definiert worden. Die Glaubwürdigkeit einer quasi-experimentellen Evaluation hängt von der Validität der Kontrollgruppen ab. Card and Krueger (1995: 23) haben einige Kriterien hierzu formuliert. Treatment- und Kontrollgruppe sollen danach einigermaßen (,reasonable“) vergleichbar sein, sich in der Vergangenheit in gemeinsamer Richtung entwickelt haben und es soll ausgeschlossen werden, dass es andere besser vergleichbare Kontrollgruppen gibt.

Für die Gebäudereinigung bot es sich an, zunächst die Auswirkungen der regional unterschiedlichen tariflichen Lohnerhöhungen bzw. Lohnsenkungen ab April 2004 für Schätzungen der Beschäftigungswirkungen von Lohnvariationen zu nutzen. Die Vergleichbarkeit war durch die Zugehörigkeit zur selben Branche höher als zu Kontrollgruppen aus jeder anderen Branche und die Entwicklung in der Vergangenheit ähnelte sich in hohem Maß. Darüber hinaus wurden als weitere externe Politikinterventionen die Aufnahme des Gebäudereiniger-Handwerks in das AEntG im Juli 2007 und die im März 2008 folgende Erhöhung der Mindestlöhne auf die Beschäftigung ausgewählt und die Wirkungen im Vergleich zu drei Kontrollgruppen aus anderen Branchen (Innenreinigung in anderen Branchen, getränkebasierte Gastronomie, Wachdienste) untersucht, die sich in der Vergangenheit nach mehreren Kriterien ähnlich wie die Gebäudereinigung entwickelt hatten.

Datenbasis der mikroökonometrischen Auswertungen war die schwach anonymisierte Stichprobe der Integrierten Arbeitsmarktbiografien (SIAB, Version 1975-2008). ${ }^{7}$ Ursprünglich waren wir davon ausgegangen, dass individuelle Stundenlöhne für einzelne Personen in der SIAB bestimmt werden könnten, indem Arbeitszeitinformationen aus dem Mikrozensus mit Entgeltinformationen aus der SIAB kombiniert würden. Dies war allerdings vor allem aufgrund zu geringer Fallzahlen - insbesondere im Mikrozensus nicht möglich. ${ }^{8}$ Der Datensatz ermöglichte jedoch, die Beschäftigungseffekte der Aufnahme in das ArbeitnehmerEntsendegesetz und der Veränderungen der vereinbarten Mindestlöhne im Vergleich zu Kontrollgruppen zu berechnen.

\footnotetext{
${ }^{7}$ Der Datenzugang erfolgte über einen Gastaufenthalt im Forschungsdatenzentrum der Bundesagentur für Arbeit im Institut für Arbeitsmarkt- und Berufsforschung (FDZ) und anschließend mittels kontrollierter Datenfernverarbeitung beim FDZ. Zum Datensatz vgl. auch Dorner u. a. (2010).

${ }^{8}$ Darüber hinaus lassen sich im Mikrozensus Beschäftigte mit der Berufskennziffer 933 (Raum-, Hausratreiniger) nicht separat identifizieren, sondern nur gemeinsam mit der Berufskennziffer 934 (Glas-, Gebäudereiniger).
}

Der Geltungsbereich der Mindestlohnregelungen in der Gebäudereinigung erstreckt sich auf gewerblich Beschäftigte. In der SIAB ist eine Unterscheidung zwischen gewerblich Beschäftigten (Arbeiter/innen) und Angestellten aber nur für Vollzeitbeschäftigte möglich. Da in der Gebäudereinigung die Mehrheit der Beschäftigten in Teilzeit oder Minijobs tätig ist, mussten wir ein anderes Vorgehen wählen, um die vom Mindestlohn betroffene Gruppe zu identifizieren.

In der Gebäudereinigung sind die Lohngruppen 1 und 6 allgemeinverbindlich. Da die deutliche Mehrheit der Beschäftigten im Gebäudereiniger-Handwerk nach Einschätzung der Sozialpartner, die auch durch die Betriebsbefragung bestätigt wurde, auf dem Niveau der untersten Lohngruppe bezahlt wird (Abb. 4), während nur wenige Beschäftigte auf dem Niveau der höheren Lohngruppen eingruppiert sind, wurde bei den ökonometrischen Auswertungen die untere Lohngruppe in den Mittelpunkt gestellt. Um diese im Datensatz zu identifizieren, haben wir die Treatmentgruppe auf Innenreiniger/innen (Berufskennziffer 933: Raum-, Hausratreiniger) in der Gebäudereinigungsbranche beschränkt, welche in der Regel gewerbliche Beschäftigte und mit hoher Wahrscheinlichkeit in die Lohngruppe 1 eingestuft sind. Geringfügig Beschäftigte in Nebenjobs konnten in den Berechnungen nicht berücksichtigt werden, weil diese erst seit 2003 in den Daten enthalten sind, und es sonst Brüche in den Zeitreihen gegeben hätte.

\subsection{Auswirkungen der regional unterschiedlichen Tariflohnänderungen 2004}

Mit dem Tarifabschluss von Oktober 2003 kam es in der Gebäudereinigung zu regional unterschiedlichen Anhebungen und Absenkungen der Tariflöhne (Gather u. a. 2005; Schlese und Schramm 2004). Für die mikroökonometrischen Schätzungen zu den Beschäftigungswirkungen der regional unterschiedlichen tariflichen Erhöhungen bzw. Absenkungen der untersten Lohngruppe 1 ab April 2004 wurden die Bundesländer je nach Ausmaß dieser Tariflohnveränderungen in fünf Bundesländergruppen eingeteilt. Die Bundesländergruppe mit den geringsten tariflichen Änderungen wurde als Referenzgruppe verwendet (Tab. 3).

Die Differenz-von-Differenzen-Schätzung mit einer dichotomen abhängigen Variable erfolgte als Random-EffectsProbit für alle Quartale der Jahre 2001 bis 2006. Hiermit wurde die Beschäftigungswahrscheinlichkeit vor dem Interventionszeitpunkt mit der Beschäftigungswahrscheinlichkeit danach verglichen. Die Tariflohnänderung erfolgte zum 1. April 2004, so dass davon auszugehen war, dass diese sich ab dem nächsten nachfolgenden Quartalsstichtag (30. Juni 2004) in den Daten bemerkbar machen konnte. Dabei haben wir die Beschäftigungswahrscheinlichkeiten im Zeitraum zwischen dem 31. März 2001 und dem 31. März 2004 mit denen zwischen dem 30. Juni 2004 und dem 31. Dezember 2006 verglichen. Es wurden demnach nicht zwei 
Tab. 3 Einteilung von Bundesländern in Gruppen

\begin{tabular}{lll}
\hline Nr. & Bundesländergruppe & $\begin{array}{l}\text { Prozentuale } \\
\text { Tariflohnänderung } \\
\text { (Lohngruppe 1) zum 1. April } \\
\end{array}$ \\
& & 2004 \\
\hline 1 & Bayern, Hessen & $-9 \%$ bis $-10,7 \%$ \\
2 & Nordrhein-Westfalen & $-6 \%$ \\
3 & Berlin, Baden-Württemberg, Rheinland-Pfalz, & $-4 \%$ bis $-4,4 \%$ \\
& Bremen, Hamburg, Niedersachsen, & \\
5 & Schleswig-Holstein & $-0,3 \%$ bis $+1,6 \%$ \\
& Saarland, Brandenburg, Thüringen (Referenzgruppe) & $+3,3 \%$ bis $+6,7 \%$ \\
\hline
\end{tabular}

Quelle: Eigene

Zusammenstellung,

Tariflohnänderung aus Gather

u. a. (2005: 31)
Nach traditionellen mikroökonomischen Annahmen wä-

Zeitpunkte miteinander verglichen, sondern der Panelcharakter der Daten genutzt und Zeiträume verglichen, wodurch die unbeobachtete Heterogenität besser kontrolliert werden konnte. Hierfür sprach auch die recht hohe Fluktuation, die teilweise dadurch bedingt ist, dass bei Verlust eines Reinigungsauftrages die Reinigungskräfte, die bislang im Objekt tätig waren, vom neuen Auftragnehmer häufig übernommen werden (Betriebsübergang). ${ }^{9}$ Der Endzeitpunkt des Beobachtungszeitraums wurde gewählt, weil bereits zum 1. Juli 2007 die Aufnahme der Gebäudereinigungsbranche in das Arbeitnehmer-Entsendegesetz erfolgte und wir diesen Zeitpunkt ausschließen wollten.

Bei den hier angewandten nicht-linearen Schätzmodellen kann die absolute Größe des geschätzten Koeffizienten nicht interpretiert werden. Sofern die interessierende unabhängige Variable (wie hier) ein Dummy ist, kann der Effekt einer Änderung dieser Dummy-Variablen auf die abhängige Variable berechnet werden. Allerdings gilt der ermittelte Effekt jeweils nur für eine spezifische Kombination von Ausprägungen der unabhängigen Variablen. Vorliegend wurden diese Effekte für spezifische Zeitpunkte und ggf. Regionen, im Übrigen jedoch für die Mittelwerte der unabhängigen Variablen (zum jeweiligen Zeitpunkt und ggf. in der jeweiligen Region) ermittelt. ${ }^{10}$ Um einschätzen zu können, wie groß der Effekt der Tariflohnänderungen war, wurden zu dem Modell auch Marginaleffekte berechnet. Dies sind die Effekte der im Vergleich zur Referenzgruppe abweichenden Tariflohnanpassungen auf die Chance einer Beschäftigung als Innenreiniger/in. Als Stichtag für diese Berechnung wurde der 30. Juni 2005 gewählt. $^{11}$

\footnotetext{
${ }^{9}$ In der Betriebsbefragung gaben über $60 \%$ der Betriebe an, dass sie im vergangenen Jahr zwischen 10 und $50 \%$ ihrer Beschäftigten neu eingestellt hatten, und fast die Hälfte der Betriebe hatten Abgänge in dieser Größenordnung. $12 \%$ der Befragten hatten sogar Abgänge von 50 bis über $100 \%$ ihrer Belegschaften (Bosch u. a. 2011: 163).

${ }^{10}$ Für die Berechnung dieser Effekte wurde die erforderliche Anpassung der geschätzten Koeffizienten vorgenommen (vgl. hierzu Arulampalam 1998).

${ }^{11}$ Für diese Berechnung wurden die Mittelwerte aller Variablen aus dem jeweiligen Regressionsmodell zum genannten Stichtag benötigt.
} re zu erwarten, dass Tariflohnsenkungen die Beschäftigung erhöhen und Tariflohnerhöhungen die Beschäftigung verringern. Die Schätzungen zu den Effekten der Tariflohnveränderungen in der Gebäudereinigungsbranche ab April 2004 bestätigen diese Erwartungen jedoch nicht. Die Ergebnisse sind nicht eindeutig und nur vereinzelt signifikant. In Nordrhein-Westfalen (Ländergruppe 2), wo die zweitstärksten Tariflohnsenkungen (um $6 \%$ ) erfolgt waren, haben sich die Beschäftigungschancen von Innenreiniger/innen deutlich und stark signifikant (um 6,3\%) verschlechtert. In der Bundesländergruppe 3 mit etwas geringeren, aber ebenfalls deutlichen Lohnabsenkungen sind die Beschäftigungschancen insgesamt demgegenüber leicht gestiegen (Tab. 4). ${ }^{12}$

Differenziert nach Beschäftigtengruppen sind nur bei den Minijobs die Beschäftigungschancen in zwei Bundesländergruppen signifikant gestiegen, was auf eine Substitution von sozialversicherungspflichtiger Beschäftigung durch Minijobs (möglicherweise begünstigt durch die Reform der Minijobs im Jahr 2003, mit der u. a. die monatliche Verdienstgrenze von 325 auf $400 €$ deutlich erhöht wurde) hindeuten könnte. Da jedoch in den anderen Beschäftigtengruppen (mit Ausnahme der Bundesländergruppe 2) keine signifikanten Rückgänge der Beschäftigungschancen in Vollzeitund Teilzeitbeschäftigung zu verzeichnen sind, wird diese These nur in der Tendenz bestätigt.

Dass die Koeffizienten in Nordrhein-Westfalen auf eine völlig andere Wirkung der Tariflohnänderung hinweisen als in den beiden anderen Bundesländergruppen mit einer Tariflohnabsenkung (die Gruppen 1 und 3), deutet auf eine

\footnotetext{
Aus Datenschutzgründen konnten uns einige Werte nicht übermittelt werden, da die Angaben auf Fallzahlen von unter 20 basierten. So hatten beispielsweise in Bayern und Hessen von 4.269 Personen unserer Stichprobe weniger als 20 einen Fachhochschulabschluss. Hier ist aber zumindest bekannt, dass die Fallzahl zwischen einem und 19 Fällen liegt. Um die Berechnung durchführen zu können, wurde hier ein Mittelwert auf der Basis von zehn Fällen angenommen. Die exakte Fallzahl würde sich erst ab der zweiten Nachkommastelle auswirken.

${ }^{12}$ Die vollständigen Modelle finden sich im Anhang I des Abschlussberichts (Bosch u. a. 2011, Tab. A.1 bis A.5).
} 
Tab. 4 Regressionsmodelle 2004, Änderung der Beschäftigungswahrscheinlichkeit durch die Tariflohnänderung (Marginaleffekte, in \%)

\begin{tabular}{|c|c|c|c|c|c|c|c|}
\hline \multicolumn{2}{|c|}{ Bundesländergruppe } & \multirow{3}{*}{$\begin{array}{l}\text { Tariflohnänderung } \\
\text { (in \%) }\end{array}$} & \multicolumn{5}{|l|}{ Modell } \\
\hline & & & \multicolumn{4}{|c|}{ Innenreiniger/innen in der Gebäudereinigungsbranche } & \multirow{2}{*}{$\begin{array}{l}\text { Innenreiniger/ } \\
\text { innen } \\
\text { insgesamt } \\
\end{array}$} \\
\hline & & & Gesamt & Vollzeit & Teilzeit & Minijobs & \\
\hline 1 & Bayern, Hessen & $-9,0$ bis $-10,7$ & $(+1,3)$ & $(-1,0)$ & $(-4,2)$ & $+\mathbf{5}, \mathbf{3}^{* *}$ & $(-0,1)$ \\
\hline 2 & Nordrhein-Westfalen & $-6,0$ & $-6,3^{* * *}$ & $-\mathbf{2 5}, \mathbf{3}^{* * *}$ & $-17,6^{* * *}$ & $(-1,0)$ & $-3,4^{* * *}$ \\
\hline 3 & $\begin{array}{l}\text { Berlin, Baden-Württemberg, } \\
\text { Rheinland-Pfalz, Bremen, } \\
\text { Hamburg, Niedersachsen, } \\
\text { Schleswig-Holstein }\end{array}$ & $-4,0$ bis $-4,4$ & $+1,9^{* *}$ & $(-1,5)$ & $(-0,5)$ & $+\mathbf{5}, \mathbf{5}^{* *}$ & $(-0,3)$ \\
\hline 4 & $\begin{array}{l}\text { Referenzgruppe: Saarland, } \\
\text { Brandenburg, Thüringen }\end{array}$ & $-0,3$ bis $+1,6$ & & & & & \\
\hline 5 & $\begin{array}{l}\text { Mecklenburg-Vorpommern, } \\
\text { Sachsen, Sachsen-Anhalt }\end{array}$ & $+3,3$ bis $+6,7$ & $(+1,2)$ & $(-5,7)$ & $(-0,6)$ & $(+4,7)$ & $+2,3^{* * *}$ \\
\hline
\end{tabular}

Quelle: SIAB, eigene Berechnung

Werte in Klammern basieren auf nicht signifikanten Koeffizienten bei einem Signifikanzniveau von $10 \%$

Zur Verdeutlichung werden zusätzlich auch die Signifikanzniveaus der Regressionskoeffizienten ausgewiesen: ${ }^{* *} p<5 \%$ und ${ }^{* * *} p<1 \%$

von den übrigen Ländergruppen abweichende regionale Entwicklung hin, die wir durch die Kontrollvariablen (insbesondere BIP pro Kopf und Arbeitslosenquoten sowie Jahresdummies) nicht auffangen konnten. Wir haben verschiedene Erklärungsansätze (darunter eine abweichende Entwicklung der Minijobs nach der Reform von 2003) geprüft, aber keinen identifizieren können, der das abweichende Ergebnis erklären könnte.

Bei der Interpretation der Ergebnisse ist zu beachten, dass sich die Gesamtwerte auf Kopfzahlen beziehen und nicht auf das Arbeitsvolumen. Da keine individuellen Informationen über die Arbeitszeit vorlagen, konnten Minijobs und sozialversicherungspflichtig Beschäftigte nicht mit der Arbeitszeit gewichtet werden und zählten somit gleich. Dies wäre kein Problem, wenn die Mehrheit der Beschäftigten Vollzeit arbeiten würden. In einer Branche wie der Gebäudereinigung mit einem hohen Anteil an sozialversicherungspflichtiger Teilzeitarbeit und geringfügiger Beschäftigung (knapp $80 \%$ ), in der zudem Verschiebungen zwischen den Beschäftigungsformen (vor allem Verringerung der geringfügigen Beschäftigung) von vielen Unternehmensleitungen gewünscht werden, sind die Werte zur Gesamtbeschäftigung hingegen mit Vorsicht zu behandeln. Da wir separate Modelle für die einzelnen Arbeitszeitformen berechnet haben, lässt sich allerdings zumindest indirekt etwas über Verschiebungen zwischen den Arbeitszeitformen sagen.

Es wurde ein zusätzliches Modell für alle Innenreiniger/innen, d.h. innerhalb und außerhalb der Gebäudereinigungsbranche, gerechnet. Die Spezifikation des Modells änderte sich nicht, lediglich die abhängige Variable wurde weiter gefasst. Diese wurde nicht nur bei einer Beschäftigung als Innenreiniger/in in der Gebäudereinigung mit eins kodiert, sondern für jede Beschäftigung als Innenreiniger/in unabhängig von der Branche, um mögliche Substitutionsbeziehungen zwischen der Innenreinigung in der Gebäudereinigungsbranche und der Reinigung in anderen Branchen zu prüfen. Die rechte Spalte von Tab. 4 verdeutlicht, dass es hierbei signifikant positive Effekte in der Bundesländergruppe 5 und negative Effekte in Nordrhein-Westfalen gab, wobei letztere in die gleiche Richtung weisen wie bei den Reinigungskräften in der Gebäudereinigungsbranche.

\subsection{Auswirkungen der Aufnahme in das Arbeitnehmer-Entsendegesetz 2007 und der Mindestlohnerhöhung 2008}

In der Schätzung für 2007/2008 wurden die Effekte der Aufnahme der Gebäudereinigungsbranche in das ArbeitnehmerEntsendegesetz ab 1. Juli 2007 und der zum 1. März 2008 folgenden Tarif- und Mindestlohnerhöhungen auf die Beschäftigungswahrscheinlichkeit als Innenreiniger/in in der Gebäudereinigungsbranche im Vergleich zu drei verschiedenen Kontrollgruppen berechnet. Dies waren Innenreiniger/innen in ausgewählten anderen Branchen sowie vergleichbare Berufsgruppen in der Gastronomie bzw. im Wach- und Sicherheitsgewerbe.

Die Innenreiniger/innen in anderen Branchen sind der Treatmentgruppe, den Innenreiniger/innen in der Gebäudereinigungsbranche, am ähnlichsten. Allerdings sind Substitutionseffekte möglich, da bei Einführung oder Erhöhung der Mindestlöhne Reinigungstätigkeiten aus der Gebäudereinigung in andere Branchen verlagert worden sein könnten. Dabei würde bei einem Rückgang der Beschäftigung der Innenreiniger/innen in der Gebäudereinigung Beschäftigung ggf. in andere Branchen verlagert. Umgekehrt könnte bei einem Anstieg der Beschäftigung der Innenreiniger/innen 
Tab. 5 Veränderung von Stundenlohn, Beschäftigtenzahl und Arbeitsvolumen zwischen 2007 und 2008, Treatment- und Kontrollgruppen (Deutschland, alle Arbeitszeitformen)

\begin{tabular}{|c|c|c|c|c|}
\hline Treatment- und Kontrollgruppen & $\begin{array}{l}\text { Änderung } \\
\text { Tariflohn }\end{array}$ & $\begin{array}{l}\text { Änderung } \\
\text { Stundenlohn }\end{array}$ & $\begin{array}{l}\text { Änderung } \\
\text { Beschäftigung }\end{array}$ & $\begin{array}{l}\text { Änderung } \\
\text { Arbeitsvolumen }\end{array}$ \\
\hline $\begin{array}{l}\text { Treatmentgruppe: } \\
\text { Innenreiniger/innen in der } \\
\text { Gebäudereinigungsbranche }\end{array}$ & $3,1 \%$ a & $2,7 \%$ & $-0,9 \%$ & $-0,5 \%$ \\
\hline $\begin{array}{l}\text { Kontrollgruppe 1: } \\
\text { Innenreiniger/innen in } \\
\text { ausgewählten anderen Branchen }\end{array}$ & n.v. & $0,2 \%$ & $-2,3 \%$ & $-1,1 \%$ \\
\hline $\begin{array}{l}\text { Kontrollgruppe 2: Köche und } \\
\text { Kellner/innen in der } \\
\text { getränkegeprägten Gastronomie }\end{array}$ & $3,2 \%^{\mathrm{a}}$ & $5,1 \%$ & $-4,5 \%$ & $-5,5 \%$ \\
\hline $\begin{array}{l}\text { Kontrollgruppe 3: Ausgewählte } \\
\text { Berufe im Wach- und } \\
\text { Sicherheitsgewerbe }\end{array}$ & $1,9 \%^{\mathrm{a}}$ & $1,9 \%$ & $2,0 \%$ & $2,7 \%$ \\
\hline
\end{tabular}

Quelle: SIAB und Mikrozensus, eigene Auswertung

${ }^{a}$ Quelle für die Tariflohnänderungen ist der Index der Tarifverdienste des Statistischen Bundesamtes. Daten waren verfügbar für das Gastgewerbe (WZ 55.0), das Wach- und Sicherheitsgewerbe (WZ 80.0) sowie die Gebäudebetreuung (WZ 81.0) nach der WZ 2008

in der Gebäudereinigungsbranche Beschäftigung aus anderen Branchen in die Gebäudereinigungsbranche verlagert worden sein. In beiden Fällen wäre die Differenz zwischen Treatment- und Kontrollgruppe größer als bei einem Vergleich zu einer Kontrollgruppe, mit der keine Substitutionsbeziehungen bestehen.

Die beiden anderen Kontrollgruppen sind den Innenreiniger/innen in der Gebäudereinigung in unterschiedlichen Aspekten ähnlich. Das Wachgewerbe ähnelt der Gebäudereinigungsbranche in seiner Kundenstruktur und hinsichtlich der Preis- und Umsatzentwicklung, hat aber eine gänzlich andere Beschäftigtenstruktur (hoher Vollzeitanteil). Die ausgewählten Berufsgruppen in der Gastronomie ähneln in der Beschäftigungsstruktur den Innenreiniger/innen in der Gebäudereinigungsbranche, während sich die Kundenstruktur durch die größere Bedeutung des privaten Konsums in der Gastronomie unterscheidet. Die Schätzung mit mehreren Kontrollgruppen zielte darauf ab, die Spezifika der Kontrollgruppen teilweise zu neutralisieren und damit zu robusteren Ergebnissen zu gelangen.

Für die Treatment- und Kontrollgruppen sind in Tab. 5 die Änderungen der tariflichen und durchschnittlichen effektiv gezahlten Stundenlöhne, die Änderung der Beschäftigung sowie die Änderung des Arbeitsvolumens zwischen 2007 und 2008 ausgewiesen. Die Tarifinformationen sind nur ein grober Anhaltspunkt für die Tarifentwicklung, da die Branchenabgrenzung nicht exakt mit der in den SIABAuswertungen verwendeten übereinstimmt. ${ }^{13}$ Weiterhin ist

\footnotetext{
${ }^{13}$ Der Index der Tarifverdienste des Statistischen Bundesamtes enthält Tarifinformationen nach der WZ 2008. Es können das Gastgewerbe (WZ 55.0), das Wach- und Sicherheitsgewerbe (WZ 80.0) sowie die Gebäudebetreuung (WZ 81.0) unterschieden werden. Wegen der unter-
}

zu beachten, dass die Tarifbindung im Gastgewerbe und im Wachgewerbe deutlich geringer ist als in der Gebäudereinigung. Die berechneten Änderungen der effektiven durchschnittlichen Stundenlöhne stimmen zumindest von ihrer Relation zueinander mit den Tariflohnänderungen überein, d.h. in der getränkegeprägten Gastronomie war die tatsächliche Lohnsteigerung am höchsten, gefolgt von der Gebäudereinigungsbranche (Treatmentgruppe) und dem Wach- und Sicherheitsgewerbe. Für die Innenreiniger/innen in ausgewählten anderen Branchen liegt keine Tarifinformation vor, da es sich um unterschiedliche Branchen handelt. Im Unterschied zum Wach- und Sicherheitsgewerbe waren die Beschäftigung und das Arbeitsvolumen in den anderen Branchen rückläufig, wobei dies in der getränkegeprägten Gastronomie deutlich stärker ausgeprägt war als in der Treatmentgruppe.

Die Schätzungen haben ergeben, dass hinsichtlich der Richtung der Wirkungen der Aufnahme in das Arbeitnehmer-Entsendegesetz und der darauf folgenden Mindestlohnerhöhung auf die Gesamtbeschäftigung (alle Arbeitszeitformen) kaum Unterschiede zwischen den Kontrollgruppen erkennbar sind (Tab. 6). Die Aufnahme in das AEntG hat sich im Vergleich zu den Kontrollgruppen 2 und 3 signifikant negativ auf die Beschäftigungswahrscheinlichkeit als Innenreiniger/in in der Gebäudereinigungsbranche ausgewirkt. Die folgende Tarif- und Mindestlohnerhöhung um 3,5\% ab 1. März 2008 hat demgegenüber bezogen auf die Kontrollgruppen 1 und 2, also im Vergleich zu den Innenreiniger/innen in ausgewählten anderen Branchen und be-

schiedlichen Wirtschaftszweigsystematik passen diese Angaben nicht exakt mit den Berechnungen auf Basis der SIAB zusammen, welche nach der WZ 2003 differenzieren. 
Tab. 6 Regression 2007/2008: Änderung der Beschäftigungswahrscheinlichkeit (Marginaleffekte, in \%)

\begin{tabular}{|c|c|c|c|c|c|}
\hline Kontrollgruppe & Intervention & Alle Arbeitszeitformen & Vollzeit & Svp Teilzeit & Minijob \\
\hline \multirow{2}{*}{$\begin{array}{l}1 \text { (Reinigungskräfte in } \\
\text { anderen Branchen) }\end{array}$} & AEntG & $(-2,7)$ & $(+0,7)$ & $+5,1^{*}$ & $-8,7^{* * *}$ \\
\hline & Mindestlohnerhöhung & $+\mathbf{8}, 0^{* * *}$ & $+11,7^{*}$ & $+14,0^{* * *}$ & $(+0,8)$ \\
\hline \multirow[t]{2}{*}{2 (Gastronomie) } & AEntG & $-5,4^{* *}$ & $-12,2^{*}$ & $(-19,7)$ & $-9,6^{* * *}$ \\
\hline & Mindestlohnerhöhung & $+\mathbf{8}, 3^{* *}$ & $(+5,7)$ & $(+14,1)$ & $+8,7^{* * *}$ \\
\hline \multirow[t]{2}{*}{3 (Wachdienste) } & AEntG & $-2,9^{* *}$ & $-7,8^{* *}$ & $-13,4^{*}$ & $(-0,03)$ \\
\hline & Mindestlohnerhöhung & $(+0,5)$ & $(+6,5)$ & $(-4,1)$ & $(-7,0)$ \\
\hline
\end{tabular}

Quelle: SIAB, eigene Berechnung

Werte in Klammern basieren auf nicht signifikanten Regressionskoeffizienten bei einem Signifikanzniveau von $10 \%$

Zur Verdeutlichung werden zusätzlich auch die Signifikanzniveaus der Regressionskoeffizienten ausgewiesen: ${ }^{*} p<10 \%$, ${ }^{* *} p<5 \%$ und *** $p<1 \%$

stimmten Berufsgruppen in der Gastronomie, positive und stark signifikante Wirkungen auf die Beschäftigungswahrscheinlichkeit als Innenreiniger/in in der Gebäudereinigung gehabt.

Die berechneten Marginaleffekte verdeutlichen zudem, dass die positiven Effekte der Tarif- und Mindestlohnerhöhungen jeweils deutlich stärker waren als die negativen Effekte der Aufnahme der Branche in das ArbeitnehmerEntsendegesetz. So hat sich die Beschäftigungswahrscheinlichkeit von Innenreiniger/innen in der Gebäudereinigung gegenüber den Innenreiniger/innen in anderen Branchen nach der tariflichen Erhöhung der Mindestlöhne stark signifikant um $8 \%$ erhöht.

Differenziert nach Intervention und Beschäftigungsformen sind markante Unterschiede erkennbar. Der Koeffizient der Aufnahme der Gebäudereinigungsbranche in das Arbeitnehmer-Entsendegesetz ist bei den vollzeitbeschäftigten Innenreiniger/innen in der Gebäudereinigung im Vergleich zu den Kontrollgruppen 2 (Gastronomie) und 3 (Wachgewerbe) signifikant negativ. Bei den sozialversicherungspflichtig teilzeitbeschäftigten Innenreiniger/innen in der Gebäudereinigung ist er im Vergleich zur Kontrollgruppe 1 schwach signifikant positiv, während im Vergleich zur Kontrollgruppe 3 schwach signifikante negative Effekte erkennbar sind. Bei den Minijobber/innen ist die Wirkung auf die Beschäftigungschancen im Vergleich zu den Kontrollgruppen 1 und 2 signifikant negativ und auch recht stark ausgeprägt. Eine mögliche Erklärung für den negativen Effekt der Aufnahme der Branche in das AEntG auf die Beschäftigungschancen in Minijobs könnte sein, dass diese Beschäftigungsform für Arbeitgeber unattraktiver geworden ist, weil evtl. vorher vorkommende Benachteiligungen der geringfügig Beschäftigten in der Gebäudereinigung nunmehr durch die Zollkontrollen wirksamer unterbunden worden sind.

Die deutlichsten Wirkungen der ab März 2008 folgenden Tarif- und Mindestlohnerhöhungen sind im Vergleich zur Kontrollgruppe 1 erkennbar. So ist die Wirkung auf die
Beschäftigungswahrscheinlichkeit von sozialversicherungspflichtig Teilzeitbeschäftigten stark signifikant positiv und mit $14 \%$ auch deutlich ausgeprägt. Auch bei den Vollzeitbeschäftigten sind positive Wirkungen zu verzeichnen, die hinsichtlich der Marginaleffekte ebenfalls recht ausgeprägt, jedoch nur schwach signifikant sind. Im Vergleich zur Kontrollgruppe 2 sind demgegenüber stark signifikante positive Wirkungen auf die Minijobs erkennbar, während bezogen auf die Kontrollgruppe 3 keinerlei signifikante Wirkungen festgestellt werden konnten. Insgesamt hat die Aufnahme der Gebäudereinigungsbranche in das AEntG offenbar einen sichtbaren Einführungseffekt gehabt, der vor allem in einer deutlichen Verringerung der Beschäftigungswahrscheinlichkeit von Beschäftigten in Minijobs bestand. Die folgende Tarif- und Mindestlohnerhöhung wirkte sich trotz möglicher Verlagerungen in die Innenreinigung in andere Branchen signifikant positiv auf die Beschäftigungswahrscheinlichkeit der Innenreiniger/innen in der Gebäudereinigungsbranche insgesamt gegenüber zwei Kontrollgruppen aus. Im Vergleich zu den Innenreiniger/innen in anderen Branchen (Kontrollgruppe 1) sind hier vor allem die Beschäftigungschancen von Teilzeitbeschäftigten gestiegen, was auf eine Umwandlung von Minijobs in sozialversicherungspflichtige Teilzeitstellen hindeuten könnte. Da die Lohnerhöhungen nur acht Monate nach der Aufnahme der Branche in das AEntG erfolgten, könnten die positiven Beschäftigungswirkungen zumindest teilweise auch noch zeitverzögerte Wirkungen des AEntG sein.

\section{Zusammenfassung und weiterer Forschungsbedarf}

Gegenstand dieses Beitrags ist die Evaluation der Mindestlohnregelungen in der Gebäudereinigung. Die Branche ist mit einem Personalkostenanteil von $72 \%$, einer hohen Markttransparenz und einer großen Vergleichbarkeit und Standardisierung der angebotenen Dienstleistungen äußerst 
wettbewerbsintensiv. Die Sozialpartner sind sich seit langem einig, durch gemeinsam vereinbarte Lohnuntergrenzen Löhne aus dem Wettbewerb zu nehmen und gleiche Wettbewerbsbedingungen für alle Unternehmen zu schaffen. Die Branche ist bereits im Juli 2007 in das AEntG aufgenommen worden. Seitdem sind die Mindestlöhne mehrfach erhöht worden. Die Grundlage für die Aufnahme des Gebäudereiniger-Handwerks in das AEntG wurde u. a. durch die Tarifvereinbarungen ab 2004 geschaffen, mit denen die regionalen Tarife mit unterschiedlicher Lohnhöhe in einen einheitlichen nationalen Tarifvertrag mit unterschiedlichen Lohnniveaus für Ost- und Westdeutschland überführt wurden. Da die Tarifverträge der Branche bereits seit den 1970er Jahren für allgemeinverbindlich erklärt worden sind, erschien fraglich, ob es überhaupt einen echten Einführungseffekt der Mindestlohnregelungen gegeben haben konnte.

Bei der Anlage der Evaluation war zu beachten, dass Reinigungstätigkeiten nicht nur von Gebäudereinigungsfirmen, sondern in anderen Branchen mit eigenen Reinigungskräften ausgeführt werden können. Ebenfalls zu berücksichtigen war angesichts des hohen Anteils von geringfügig und sozialversicherungspflichtig Teilzeitbeschäftigten, dass sich Beschäftigungswirkungen auch in der Veränderung des Arbeitsvolumens ausdrücken können.

Die statistischen Voraussetzungen für die Evaluation der Mindestlohnregelungen waren alles andere als ideal. So fehlten präzise Daten zur Arbeitszeit und zu den Stundenlöhnen der Beschäftigten in der Gebäudereinigungsbranche ebenso wie für die verschiedenen Kontrollgruppen. Hierzu konnten zwar Angaben zur Arbeitszeit aus dem Mikrozensus mit Angaben zur Beschäftigung aus der Stichprobe Integrierter Arbeitsmarktbiographien (SIAB) kombiniert werden. Angesichts der großen Unterschiede zwischen beiden Datensätzen z. B. zur Zahl der Beschäftigten in der Gebäudereinigungsbranche insgesamt und in den Reinigungsberufen und auch den teils geringen Fallzahlen im Mikrozensus für einzelne Bundesländer war dies weniger differenziert möglich als ursprünglich angenommen.

Aufgrund dessen waren auch keine belastbaren Aussagen zu den genauen Auswirkungen der Einführung und der Erhöhung der Mindestlöhne auf die tatsächliche Bezahlung möglich. Durch unsere repräsentative Betriebsbefragung konnten wir dies nur teilweise kompensieren, da Fragen, die sich auf die eventuelle Missachtung gesetzlicher Vorschriften beziehen, nicht zu belastbaren Ergebnissen führen. Allerdings ließen sich durch die Kombination der Betriebsbefragung mit einer Betriebsrätebefragung und Betriebsfallstudien, deren Ergebnisse alle in die gleiche Richtung wiesen, Trends identifizieren.

Die meisten Beschäftigten der Branche sind in der Unterhalts- und Innenreinigung tätig und werden nach dem unteren Mindestlohn für die Innenreinigung bezahlt, den man daher als die „going rate“ in der Branche bezeichnen kann. Der Anteil der Beschäftigten in der Außenreinigung ist insgesamt gering, so dass in den meisten Betrieben nur wenige Beschäftigte Anspruch auf den höheren Mindestlohn haben. Die Mindestlöhne werden sowohl von den Sozialpartnern als auch den Betrieben und Betriebsräten als unerlässlich angesehen, um verbindliche Lohnuntergrenzen zu sichern, die im Verdrängungswettbewerb der Branche sonst zur Disposition stehen würden. Ohne die Mindestlohnregelungen würden sich die Löhne nach Ansicht der befragten Unternehmen und Betriebsräte auf einem deutlich niedrigeren Niveau einpendeln, was dafür spricht, dass diese positive Effekte auf den Arbeitnehmerschutz haben.

Die Einhaltung der Mindestlöhne nach dem AEntG wird im Vergleich zur vorherigen Allgemeinverbindlichkeit der tariflichen Regelungen als deutlich effektiver angesehen. Dies wird im Wesentlichen auf die Zollkontrollen und stärkeren Sanktionen zurückgeführt, aber auch auf die durchgreifende Mithaftung der Auftraggeber sowie die bessere Informiertheit der Beschäftigten und ihren erhöhten Durchsetzungswillen. Indizien für eine direkte Unterschreitung der Mindestlöhne gibt es nur vereinzelt, während die Verbreitung von Strategien zur legalen oder illegalen Kompensation von Lohnerhöhungen teilweise unterschiedlich eingeschätzt wird und ihre Wirkungen auf den Schutz der Arbeitnehmer/innen nicht immer eindeutig bestimmbar sind. Zeitarbeit wird von etwa jedem fünften Betrieb genutzt, während befristete Arbeitsverträge in knapp $40 \%$ der Betriebe zum Einsatz kommen. Dabei gibt es sowohl Betriebe mit einem Befristungsanteil von weniger als $20 \%$ als auch in Einzelfällen Befristungsquoten von über $80 \%$.

Die Betriebe schreiben der Aufnahme der Gebäudereinigungsbranche in das AEntG weitaus häufiger positive oder neutrale Wettbewerbswirkungen zu als negative. Als wichtiger positiver Wettbewerbsfaktor wurde die Herstellung von Wettbewerbsgleichheit durch eine wirkungsvolle Lohnuntergrenze genannt. Die positive Einschätzung dieser Wettbewerbsfunktion der Mindestlöhne beruht vor allem auf der Wahrnehmung einer effektiveren Kontrolle der Einhaltung der Mindestlöhne. Teilweise wurde auch auf ein verbessertes Image der Branche hingewiesen, das auch die Personalrekrutierung erleichtere. Von den Sozialpartnern und auch in manchen Betriebsfallstudien wurde darauf verwiesen, dass die Mithaftung der Auftraggeber ein wichtiger Faktor sei, der die Einhaltung der Mindestlöhne nach Aufnahme in das AEntG erheblich verbessert habe. In der Betriebsbefragung wurde demgegenüber deutlich, dass mehr als die Hälfte der Betriebe meinten, dass die Stundenverrechnungssätze nicht angemessen gesteigert werden konnten. Dies könnte darauf verweisen, dass weiterhin Bedarf besteht, Kunden darüber zu informieren, wie hoch realistische Verrechnungssätze für Reinigungsdienstleistungen unter Berücksichtigung der Mindestlöhne sein müssen. 
Die Entwicklung der Zahl der Beschäftigten und des Arbeitsvolumens ließ keine negativen Effekte der Aufnahme des Gebäudereiniger-Handwerks in das AEntG und der folgenden Erhöhung der Mindestlöhne erkennen. Feststellbar waren nur leichte konjunkturelle Schwankungen. Die Gebäudereinigungsbranche konnte zwischen 2000 und 2008 das Arbeitsvolumen deutlich (um knapp $25 \%$ ) steigern, während das Arbeitsvolumen der Reinigungskräfte in anderen Branchen leicht gesunken ist.

Bei den mikroökonometrischen Schätzungen der Beschäftigungswirkungen haben wir zum einen die Auswirkungen der regional unterschiedlichen tariflichen Lohnerhöhungen bzw. Lohnsenkungen ab April 2004 untersucht. Die Vergleichbarkeit war durch die Zugehörigkeit zur selben Branche höher als zu Kontrollgruppen aus jeder anderen Branche und die Entwicklung in der Vergangenheit ähnelte sich in hohem Maß. Zum anderen wurden als weitere externe Politikinterventionen die Aufnahme des Gebäudereiniger-Handwerks in das AEntG im Juli 2007 und die im März 2008 folgende Erhöhung der Mindestlöhne auf die Beschäftigung genutzt, um die Wirkungen im Vergleich zu drei Kontrollgruppen aus anderen Branchen (Innenreinigung in anderen Branchen, Gastronomie, Wachdienste), die sich in der Vergangenheit nach unterschiedlichen Kriterien ähnlich wie die Gebäudereinigung entwickelt haben, zu untersuchen.

Unsere Schätzungen haben ergeben, dass die regional unterschiedlichen Tariflohnveränderungen in der Lohngruppe $1 \mathrm{im}$ Jahre 2004 keinen eindeutigen Einfluss auf die Beschäftigungschancen der Innenreiniger/innen in der Gebäudereinigung hatten. Signifikante Wirkungen ließen sich nur in zwei Ländergruppen feststellen, in denen sich die Beschäftigungswahrscheinlichkeit in Minijobs erhöht hatte, was für eine Substitution sozialversicherungspflichtiger Beschäftigung sprechen könnte. Wir haben weiterhin eine Sonderentwicklung in NRW registriert, deren Ursachen sich mit den zur Verfügung stehenden Indikatoren nicht identifizieren ließen.

Die Ergebnisse der Schätzungen zu den Auswirkungen der Aufnahme der Branche in das AEntG und der folgenden Erhöhung der Mindestlöhne lassen sich wie folgt zusammenfassen: Die Aufnahme der Branche in das Arbeitnehmer-Entsendegesetz im Juli 2007 hat die Beschäftigungswahrscheinlichkeit der Innenreiniger/innen in der Gebäudereinigung im Vergleich zu zwei Kontrollgruppen verringert. Die anschließende Tarif- und Mindestlohnerhöhung ab März 2008 hat die Beschäftigungschancen demgegenüber im Vergleich zu zwei Kontrollgruppen signifikant erhöht. Die Berechnung der Marginaleffekte hat gezeigt, dass die positiven Wirkungen der Tarif- und Mindestlohnerhöhungen deutlich höher waren als die negativen Effekte der Aufnahme in das AEntG, so dass die positiven Wirkungen insgesamt überwogen.
Die Differenzierung nach Arbeitszeitformen hat ergeben, dass die Aufnahme der Branche in das AEntG im Vergleich zu den Innenreiniger/innen in anderen Branchen, die der Treatmentgruppe am ähnlichsten sind, nur die Beschäftigungschancen von geringfügig Beschäftigten stark signifikant verschlechterte. Möglicherweise - und diese Schlussfolgerung unterstützten auch die Interviews in den Fallstudienbetrieben - ist geringfügige Beschäftigung für Arbeitgeber mit der Aufnahme in das AEntG unattraktiver geworden, weil evtl. vorher vorkommende Benachteiligungen der geringfügig Beschäftigten in der Gebäudereinigung nunmehr durch die Zollkontrollen wirksamer unterbunden werden. Die Schätzergebnisse für die folgenden Tarif- und Mindestlohnerhöhungen ab März 2008 sprechen dafür, dass Minijobs insbesondere durch sozialversicherungspflichtige Teilzeitbeschäftigung substituiert worden sind. Da die Lohnerhöhungen nur acht Monate nach der Aufnahme der Branche in das AEntG erfolgten, könnten die positiven Beschäftigungswirkungen zumindest teilweise auch noch zeitverzögerte Wirkungen des AEntG sein. Auch unsere deskriptiven Auswertungen haben gezeigt, dass Vollzeit- und Teilzeitbeschäftigung auf Kosten der Minijobs zugenommen haben und das Arbeitsvolumen in den letzten Jahren gestiegen ist.

Geringfügig Beschäftigte in Nebenjobs konnten in den Schätzungen nicht berücksichtigt werden, weil diese erst seit 2003 in den Daten enthalten sind, und es sonst Brüche in den Zeitreihen gegeben hätte. Da der Anteil der geringfügig Nebenbeschäftigten in der Gebäudereinigungsbranche seit 2003 deutlich gestiegen ist und im Jahr 2010 bei über $21 \%$ aller Beschäftigten lag, ist nicht auszuschließen, dass ihre Nichtberücksichtigung die Schätzergebnisse beeinflusst hat. Allerdings spricht deren zunehmende Zahl dafür, dass die Beschäftigungswirkungen bei Einbeziehung der Nebenbeschäftigten eher positiver ausgefallen wären.

Bei der Evaluation der Mindestlohnregelungen in der Gebäudereinigung mussten gravierende Datenmängel berücksichtigt werden. Dass sich dieses Datenproblem, das vor allem in der mangelnden Erfassung der Arbeitszeiten von Teilzeitbeschäftigten (sozialversicherungspflichtig und geringfügig) in der amtlichen Statistik besteht, in absehbarer Zeit lösen lässt, erscheint eher unwahrscheinlich. Insofern stellt sich die Frage, auf welchem Weg Abhilfe geschaffen werden könnte. Eine repräsentative Befragung von Beschäftigten in der Gebäudereinigung vor allem mit Fragen zu den tatsächlich gezahlten Löhnen und den Arbeitszeiten könnte dazu beitragen, diese Lücke möglichst zeitnah zu schlieBen. Eine solche Befragung könnte auch andere Mindestlohnbranchen sowie vergleichbare Kontrollgruppen (Branchen oder Gruppen wie z. B. Reinigungskräfte in anderen Branchen) einbeziehen. Denkbar wäre auch ein umfassendes „Mindestlohn-Panel“, mit dem Beschäftigte und Betriebe in regelmäßigen Zeitabständen befragt werden. Dies wür- 
de eine fortlaufende Beobachtung der Einhaltung von Mindestlöhnen sowie eine umfassendere Analyse ihrer Auswirkungen ermöglichen.

\section{Kurzfassung}

Die Gebäudereinigungsbranche ist wegen der großen Vergleichbarkeit und Standardisierung der angebotenen Dienstleistungen äußerst wettbewerbsintensiv. Aufgrund des hohen Anteils der Personalkosten von rund $72 \%$ ist die Lohnhöhe zum wichtigsten Parameter im Preiswettbewerb der Branche geworden. Die Sozialpartner wollten mit der Vereinbarungen von Mindestlöhnen verbindliche Untergrenzen für den Wettbewerb setzen. Im Unterschied zu den meisten anderen Mindestlohnbranchen gibt es in der Gebäudereinigung zwei unterschiedlich hohe Mindestlöhne für die Innenund Außenreinigung. Die Branche wurde im Juli 2007 in das Arbeitnehmer-Entsendegesetz (AEntG) aufgenommen. Die Grundlage hierfür wurde $u$. a. durch die Tarifvereinbarungen ab 2004 geschaffen, in denen die zuvor unterschiedlichen regionalen Tarife in einen einheitlichen Tarifvertrag mit unterschiedlichen Lohnniveaus für Ost- und Westdeutschland überführt wurden. Da die Tarifverträge der Branche bereits seit den 1970er Jahren für allgemeinverbindlich erklärt worden waren, erschien vorab fraglich, ob es überhaupt einen echten Einführungseffekt gegeben haben konnte.

Die Evaluation basiert auf Auswertungen amtlicher Datenquellen, einer repräsentativen Unternehmensbefragung, einer Betriebsrätebefragung, von Experteninterviews und Fallstudien und mikroökonometrischen Berechnungen auf der Basis der Stichprobe Integrierter Arbeitsmarktbiographien (SIAB) mit mehreren Kontrollgruppen. Die Wirkungen der Mindestlohnregelungen auf die Entlohnung und die Eingriffsintensität konnten mikroökonometrisch nicht ermittelt werden, weil rund $80 \%$ der Beschäftigten in der Gebäudereinigungsbranche in Teilzeit oder Minijobs arbeiten und keine präzisen Angaben zu deren Arbeitszeit vorlagen. Die angedachte Kombination von Verdienstangaben aus der SIAB und Arbeitszeitangaben aus dem Mikrozensus erwies sich angesichts der großen Unterschiede z. B. zur Zahl der Beschäftigten in den Reinigungsberufen und der teils geringen Fallzahlen im Mikrozensus als unzuverlässig. Die repräsentative Betriebsbefragung und die qualitativen Interviews legen jedoch nahe, dass der untere Mindestlohn für die Innenreinigung die ,going rate“ in der Branche ist, während der höhere Mindestlohn für die Außenreinigung quantitativ eine wesentlich geringere Bedeutung hat.

Die befragten Expert/innen, Betriebsräte und Unternehmen stimmen in der Einschätzung überein, dass die Mindestlöhne deutlich stärker eingehalten werden als die allgemeinverbindlichen Tariflöhne in den Jahren zuvor. Dies wird im Wesentlichen auf die Zollkontrollen und stärkeren Sanktionen zurückgeführt, aber auch auf die durchgreifende Mithaftung der Auftraggeber sowie die bessere Informiertheit der Beschäftigten. Indizien für eine direkte Unterschreitung der Mindestlöhne gibt es nur vereinzelt, während die Verbreitung von Strategien zur legalen oder illegalen Kompensation von Lohnerhöhungen teilweise unterschiedlich eingeschätzt wird, und ihre Wirkungen auf den Schutz der Arbeitnehmer/innen nicht eindeutig bestimmbar sind. Dass in der Betriebsbefragung mehr als die Hälfte der Betriebe meinten, dass die Stundenverrechnungssätze nicht angemessen gesteigert werden konnten, verweist darauf, wie schwierig es ist, in einem so wettbewerbsintensiven Markt realistische Verrechnungssätze für Reinigungsdienstleistungen durchzusetzen.

Die deskriptiven Analysen zur Entwicklung der Zahl der Beschäftigten und des Arbeitsvolumens ließen keine negativen Effekte der Mindestlohnregelungen erkennen. Feststellbar waren nur leichte konjunkturelle Schwankungen. Die Gebäudereinigungsbranche konnte zwischen 2000 und 2008 das Arbeitsvolumen deutlich (um knapp $25 \%$ ) steigern, während das Arbeitsvolumen der Reinigungskräfte in anderen Branchen leicht gesunken ist. Zwischen 2003 und 2010 ist die Zahl der Beschäftigten um fast $9 \%$ gestiegen und im Vergleich zu 2005 sogar um 10,5\%. Dieser Zuwachs ging fast ausschließlich auf eine Zunahme sozialversicherungspflichtiger Beschäftigung zurück, die zwischen 2005 und 2010 um gut $22 \%$ gewachsen ist.

Die Beschäftigungswirkungen wurden mit dem Kontrollgruppenansatz analysiert. Dabei wurden die Wirkungen zum einen für die regional unterschiedlichen tariflichen Erhöhungen und Absenkungen der damals allgemeinverbindlichen Lohngruppe 1 ab April 2004 untersucht. Für diese Schätzungen wurden die Bundesländer nach dem prozentualen Ausma $ß$ der tariflichen Lohnveränderungen in der untersten Lohngruppe in fünf Gruppen unterteilt. Als Kontrollgruppe wurde diejenige verwendet, in der die Löhne kaum verändert worden waren. Die Ergebnisse zeigen, dass die regional unterschiedlichen Tariflohnveränderungen in der Lohngruppe 1 im Jahre 2004 keinen eindeutigen Einfluss auf die Beschäftigungschancen als Innenreiniger/innen in der Gebäudereinigung hatten.

Zum anderen wurden Schätzungen zu den Auswirkungen der Aufnahme der Branche in das AEntG und der folgenden Erhöhung der Mindestlöhne mit drei Kontrollgruppen durchgeführt. Diese haben ergeben, dass die Aufnahme in das AEntG im Juli 2007 die Beschäftigungswahrscheinlichkeit als Innenreiniger/innen in der Gebäudereinigung im Vergleich zu zwei Kontrollgruppen verringert hat. Die anschließende Tarif- und Mindestlohnerhöhung ab März 2008 hat die Beschäftigungschancen demgegenüber im Vergleich zu zwei Kontrollgruppen signifikant erhöht. Die Berechnung der Marginaleffekte ergab, dass die positiven Wirkun- 
gen überwogen. Die Differenzierung nach Arbeitszeitformen hat ergeben, dass die Aufnahme der Branche in das AEntG im Vergleich zu den Innenreiniger/innen in anderen Branchen, die der Treatmentgruppe am ähnlichsten sind, nur die Beschäftigungschancen von geringfügig Beschäftigten stark signifikant verschlechterte. Möglicherweise und diese Schlussfolgerung unterstützten auch die qualitativen Interviews - ist geringfügige Beschäftigung für Arbeitgeber mit der Aufnahme in das AEntG unattraktiver geworden, weil evtl. vorher vorkommende Benachteiligungen der geringfügig Beschäftigten nunmehr durch die Zollkontrollen wirksamer unterbunden werden. Die Schätzergebnisse für die Tarif- und Mindestlohnerhöhungen ab März 2008 sprechen dafür, dass Minijobs insbesondere durch sozialversicherungspflichtige Teilzeitbeschäftigung substituiert worden sind. Da die Lohnerhöhungen nur acht Monate nach der Aufnahme der Branche in das AEntG erfolgten, könnten die positiven Beschäftigungswirkungen teilweise auch noch zeitverzögerte Wirkungen des AEntG sein.

\section{Executive summary}

Because of the high degree of comparability and standardisation of the services provided, the commercial cleaning industry is highly competitive. The high share of labour costs, at around $72 \%$, means that wage levels have become the most important parameter in price competition in the industry. By concluding agreements on minimum wages, the social partners have sought to set binding lower limits on competition. In contrast to most other minimum wage sectors, there are two different minimum wages in the commercial cleaning industry, one for interior and one for exterior cleaning. In July 2007, coverage by the Posted Workers Act was extended to the cleaning industry. The basis for this was created, among other things, by the collective agreements concluded from 2004 onwards, in which the previously separate regional wage agreements were converted into a single agreement with different wage levels for Eastern and Western Germany. Since the collective agreements in the industry had already been declared generally binding since the 1970s, it initially appeared questionable whether there could have been any real introduction effect at all.

The evaluation is based on analyses of official data sources, a representative company survey, a survey of works councils, expert interviews and case studies and microeconomic estimations based on the Sample of Integrated Labour Market Biographies (SIAB) with several control groups. The effects of the minimum wage regulations on pay and work intensity could not be ascertained by microeconometric methods, because around $80 \%$ of workers in the commercial cleaning sector are employed part-time or in 'mini-jobs' and no precise data on their working time was available. The planned combination of earnings data from the SIAB and working time data from the micro-census proved to be unreliable because of the considerable differences in, for example, the number of employees in the cleaning occupations and the small numbers of cases in some of the micro-census data. However, the representative company surveys and the qualitative interviews suggest that the lower minimum wage for interior cleaning constitutes the 'going rate' in the sector, while the higher minimum wage for exterior cleaning is of considerably less significance in quantitative terms.

The experts, works councillors and companies that were questioned agreed with the assessment that compliance with the minimum wages was considerably greater than with the generally binding wage rates that had prevailed previously. This is attributed essentially to monitoring by German $\mathrm{Cu}-$ stoms and stronger sanctions, as well as to the deep commitment to compliance displayed by contracting bodies and greater knowledge on the part of employees. Only in isolated cases is there any evidence of direct undercutting of minimum wages, while assessments of the extent to which employers adopt strategies to offset wage increases by legal or illegal means differ somewhat and their effects on employee protection cannot be clearly determined. The fact that more than half of the companies surveyed declared that their hourly charges could not be adequately increased indicates how difficult it is to charge realistic rates for cleaning services in such a competitive market.

The descriptive analyses of the evolution of employment levels and working time volumes do not reveal any negative effects induced by the minimum wage regulations. Only slight cyclical fluctuations could be detected. Between 2000 and 2008, the volume of working time in the commercial cleaning industry increased considerably by just under $25 \%$, while the volume of hours worked by cleaning workers in other sectors fell slightly. Between 2003 and 2010, the number of employees rose by almost $9 \%$ and in comparison with 2005 by as much as $10.5 \%$. This growth was due almost solely to an increase in the number of jobs liable for social security contributions, which grew by a good $22 \%$ between 2005 and 2010.

The employment effects were analysed using the control group approach. Firstly, the effects of the increases and reductions in the then generally binding pay grade 1 from April 2004 onwards, which varied from Land to Land, were investigated. For the purpose of these estimations, the Länder were divided into five groups according to the percentage change in the collectively agreed rates in the bottom pay grade. The control group was made up of those Länder in which wages had scarcely changed. The results show that the regionally variable changes in pay grade 1 that came into effect in 2004 had no clear influence on the employment 
opportunities as interior cleaning workers in the commercial cleaning sector.

Secondly, the effects of the application of the Posted Workers Act and the consequent increase in minimum wage rates were estimated using three control groups. These estimates showed that the application of the Posted Workers Act in July 2007 reduced the probability of finding work as interior cleaning worker in the commercian cleaning sector relative to two control groups. In contrast, the subsequent increase in pay rates and the minimum wage from March 2008 onwards significantly increased the likelihood of finding employment in comparison with two control groups. Estimation of the marginal effects showed that the positive effects predominated. Differentiation by form of working time showed that the application to the industry of the Posted Workers Act had a strongly significant negative effect only on the employment chances of 'mini-jobbers' compared with interior cleaning workers in the sectors most similar to that of the treatment group. It may be - and this conclusion is also supported by the qualitative interviews - that the application of the Posted Workers Act has made marginal part-time work less attractive to employers because any disadvantaging of 'mini-jobbers' that may have taken place earlier is now being more effectively prevented by the monitoring activities of the German Customs. The results of the estimates for the increases in pay rates and minimum wages introduced from March 2008 onwards suggest that mini-jobs in particular have been replaced by part-time jobs liable for social security contributions. Since the wage increases were implemented only eight months after application of the Posted Workers Act to the industry, the positive employment effects may also be attributable in part to delayed effects of the Act.

\section{Literatur}

Arulampalam, W.: A note on estimated coefficients in random effects probit models (1998). Oktober 1998. University of Warwick, Warwick (UK). http://www2.warwick.ac.uk/fac/ soc/economics/research/workingpapers/publications/renote5.pdf

Bispinck/WSI-Tarifarchiv: Tarifpolitischer Jahresbericht 2009: Tarifverdienste trotz Krise mit kräftigem Plus - Effektivverdienste erneut im Minus, Düsseldorf: Hans-Böckler-Stiftung, http://www.boeckler.de/pdf/p_ta_jb_2009.pdf (2010)

BIV (Bundesinnungsverband des Gebäudereiniger-Handwerks): Bedeutung der Aufnahme in Das Entsendegesetz Für die Kunden Von Gebäudereinigungsdienstleistungen. Kundeninformation, Bonn (2007)

BIV (Bundesinnungsverband des Gebäudereiniger-Handwerks): Daten und Fakten zum Gebäudereiniger-Handwerk, Bonn (2011a)

BIV (Bundesinnungsverband des Gebäudereiniger-Handwerks): Neue Tarifverträge 2012, Bonn. http://www.gebaeudereiniger.de/index. php $?$ id $=1175 \&$ type $=0 \&$ jumpurl $=$ uploads $\% 2$ Fmedia $\%$ 2FHinweise_zur_Lohngruppe_6_und_6a_LTV.pdf (2011b)

BIV (Bundesinnungsverband des Gebäudereiniger-Handwerks): Kalkulation in der Gebäudereinigung. Beispielberechnung von Stundenverrechnungssätzen. Unterhalts- bzw. Innenreinigung. Lehrmaterial. Ausgabe vom 10. Januar 2011, Bonn (2011c)
BIV (Bundesinnungsverband des Gebäudereiniger-Handwerks): Daten und Fakten. Betriebszahlen, Beschäftigte und Umsatz, Bonn. http://www.gebaeudereiniger.de/daten_fakten.html (2012)

Bosch, G., Kalina, T., Kern, C., Neuffer, S., Schwarzkopf, M., Weinkopf, C., Evaluation bestehender gesetzlicher Mindestlohnregelungen - Branche: Gebäudereinigung. Abschlussbericht. Forschungsauftrag des Bundesministeriums für Arbeit und Soziales. Duisburg: Institut Arbeit und Qualifikation. http://www.bmas.de/SharedDocs/Downloads/DE/ PDF-Meldungen/evaluation-mindestlohn-gebaedereinigung. pdf?_blob=publicationFile (2011)

Card, D., Krueger, A.B.: Myth and Measurement: The New Economics of the Minimum Wage. Princeton University Press, Princeton (1995)

Deutscher Sparkassenverlag: Reinigungsbetriebe. WZ-Code 74.7. BranchenReport 2009. Stuttgart (2009)

Dorner, M., Heining, J., Jacobebbinghaus, P., Seth, S.: Stichprobe der Integrierten Arbeitsmarktbiografien (SIAB) 1975-2008. FDZ-Datenreport 1/2010. Forschungsdatenzentrum der Bundesagentur für Arbeit im Institut für Arbeitsmarkt- und Berufsforschung, Nürnberg (2010)

Gather, C., Gerhard, U., Schroth, H., Schürmann, L.: Vergeben und vergessen? Gebäudereinigung im Spannungsfeld zwischen kommunalen Diensten und Privatisierung. VSA, Hamburg (2005)

Grömling, M.: Branchenporträt Gebäudereiniger-Handwerk, Bonn (2010)

Körner, T., Puch, K.: Der Mikrozensus im Kontext anderer Arbeitsmarktstatistiken. Ergebnisunterschiede und ihre Hintergründe. Wirtsch. Stat. 6, 528-552 (2009)

Promberger, M.: Typenbildung mit quantitativen daten. Methodologische Überlegungen. IAB-Discussion Paper 12/2011, Nürnberg: Institut für Arbeitsmarkt- und Berufsforschung (2011)

Schlese, M., Schramm, F.: In: Implikationen der Tarifverträge zur Leiharbeit für die Tarif- und Beschäftigungsbedingungen im Gebäudereiniger-Handwerk. Berichte der Werkstatt für Organisations- und Personalforschung Nr. 15, Berlin (2004)

Statistisches Bundesamt (o. J.): Strukturerhebung im Dienstleistungsbereich. Verschiedene Jahrgänge. Datenlieferung an das IAQ. Wiesbaden

Zoll: Bundesweite Schwerpunktprüfung in der Gebäudereinigungsbranche. Pressemitteilung vom 20. Dezember 2010, Berlin (2010)

Gerhard Bosch Studium der Volkswirtschaftslehre und Soziologe an der Universität Köln, 1972 Abschluss als Diplom-Volkswirt und Soziologe. 1973-1977 wissenschaftlicher Mitarbeiter an der Sozialforschungsstelle in Dortmund und 1977 Promotion an der Universität Dortmund. Von 1977 bis 1980 wissenschaftlicher Mitarbeiter an der Universität Bielefeld und 1981 bis 1989 wissenschaftlicher Referent im Wirtschafts- und Sozialwissenschaftlichen Institut (WSI). 1989 bis 2006 Abteilungsleiter und seit 2002 Vizepräsident des Instituts Arbeit und Technik, Gelsenkirchen. 1992 Habilitation an der Universität Osnabrück und seit 1993 Professor für Soziologie an der Universität Duisburg. Seit 2007 Geschäftsführender Direktor des Instituts Arbeit und Qualifikation (IAQ), Universität Duisburg-Essen.

Forschungsgebiete: Arbeitsmarkt- und Beschäftigungspolitik, Berufliche Aus- und Weiterbildung, Industrielle Beziehungen, Arbeitszeit, Personalpolitik.

gerhard.bosch@uni-due.de

Thorsten Kalina Studium der Sozialwissenschaften an der RuhrUniversität Bochum und Abschluss als Diplom-Sozialwissenschaftler 1999. Von 2000 bis 2006 wissenschaftlicher Mitarbeiter im Institut Arbeit und Technik, Gelsenkirchen, und seit Anfang 2007 im Institut Arbeit und Qualifikation (IAQ), Universität Duisburg-Essen. Promotion 2012 an der Universität Duisburg-Essen. 
Forschungsgebiete: Arbeitsmarkt- und Beschäftigungspolitik, Quantitative Methoden, Niedriglöhne, Aufstiegsmobilität.

thorsten.kalina@uni-due.de

Claudia Weinkopf Studium der Wirtschafts- und Sozialwissenschaften an der Universität Dortmund mit Abschluss als Diplom-Volkswirtin 1989. Promotion 1996 an der Universität Dortmund. Von 1990 bis 2006 wissenschaftliche Mitarbeiterin bzw. Forschungsdirektorin im In- stitut Arbeit und Technik (IAT), Gelsenkirchen. Seit 2007 Stellvertretende Geschäftsführende Direktorin des Instituts Arbeit und Qualifikation (IAQ), Universität Duisburg-Essen, und Leiterin der Forschungsabteilung „Flexibilität und Sicherheit“.

Forschungsgebiete: Arbeitsmarkt- und Beschäftigungspolitik, Industrielle Beziehungen, Personalpolitik, Prekäre Beschäftigung, Gender. claudia.weinkopf@uni-due.de 